Pacific

Journal of

Mathematics

RATIONAL HOMOTOPY OF CIRCLE ACTIONS

Volker HaUschild

Volume $191 \quad$ No. 2

December 1999 


\title{
RATIONAL HOMOTOPY OF CIRCLE ACTIONS
}

\author{
VOLKER HAUSCHILD
}

We classify circle actions on $F$-spaces $X$ with $H^{o d}(X ; \mathbb{Q})=$ 0 up to equivariant rational homotopy equivalence by the concept of g-points in the spectrum of the cohomology ring of $B_{\text {aut }_{0}}(X)$. In the case of circle actions on closed oriented manifolds $X$ with the rational cohomology of flag varieties $K / T$ this gives a generalization of the rational homotopy classification of linear (or homomorphic) circle actions to nonlinear $S^{1}$-actions.

\section{Introduction.}

There is an increasing experience that rational methods in classification of maps between classifying spaces and homogeneous spaces respectively, can be very effective thanks to the rigidity of spaces defined by compact Lie groups. A fruitful application of rational methods can be found in the theory of transformation groups. The main purpose of the present note is to show how the theory of classification of linear actions of compact Lie groups on homogeneous spaces can be embedded into a more general theory including also nonlinear actions. Suppose $G$ to be a compact connected Lie group and let $\phi: G \times X \rightarrow X$ an action on a homogeneous space $X=K / U$, where $U \subset K$ is a equal rank pair of compact Lie groups. Then $\phi$ is called linear if there exists a continuous homomorphism $\rho: G \rightarrow K$ with $\phi=\phi_{\rho}, \phi_{\rho}(g, k U)=\rho(g) k U$. In the case $U$ a maximal torus not only the equivariant rational homotopy type but also the stronger $G$-homotopy type and even the diffeomorphism type of such an action is essentially given by the character of $\rho$, see Thm. 10 .

In rational homotopy theory a 1 -connected space $X$ is called of $F$-type if the rational homotopy and the rational cohomology are finite $\mathbb{Q}$-vector spaces. If in addition the condition $H^{o d}(X ; \mathbb{Q})=0$ is satisfied it has been shown in $[6]$ that $H^{*}(X ; \mathbb{Q})$ is a complete intersection. Let $B \operatorname{aut}_{0} X$ be the classifying space for the functor of fiber homotopy types of oriented Hurewicz fibrations with fiber homotopy equivalent to $X$. Then it has been conjectured by S. Halperin that $H^{o d}\left(\operatorname{Baut}_{0}(X) ; \mathbb{Q}\right)=0$. This conjecture has been proved in a series of cases, so for example if $X$ is a homogeneous space $K / U, r k K=$ $r k U$, see e.g., [15]. Suppose $G$ operates continuously on $X$ by an action $\varphi$. Then $\varphi$ induces a homomorphism of topological monoids $\alpha: G \rightarrow \operatorname{aut}(X)$, 
where $\operatorname{aut}(X)$ is the topological monoid of self homotopy equivalences of $X$. This in turn induces a map $B \alpha: B_{G} \rightarrow B$ aut $(X)$ of classifying spaces which gives rise to a homomorphism $H^{*}(B \alpha): H^{*}(B \operatorname{aut}(X) ; \mathbb{Q}) \rightarrow H^{*}\left(B_{G} ; \mathbb{Q}\right)$. This is the invariant we are mainly considering in this note. If $G$ is acting linearly or homomorphically on $U(N) / T(N)$ the homomorphism factorizes over the natural homomorphism $H^{*}(\operatorname{Baut}(X) ; \mathbb{Q}) \rightarrow H^{*}\left(B_{U(N)} ; \mathbb{Q}\right)$ and thus gives a homomorphism $H^{*}\left(B_{U(N)} ; \mathbb{Q}\right) \rightarrow H^{*}\left(B_{G} ; \mathbb{Q}\right)$ which essentially is nothing else than the total Chern class of the principal bundle

$$
U(N) \rightarrow E_{G} \times_{G} U(N) \rightarrow B_{G} .
$$

But this in turn classifies the rational fiber homotopy type of the corresponding flag bundle

$$
U(N) / T^{N} \rightarrow E_{G} \times_{G}\left(U(N) / T^{N}\right) \rightarrow B_{G} .
$$

Therefore the homomorphism $H^{*}(B \alpha)$ can be taken into consideration as an object generalizing representation theory to the case in which $G$ does not act linearly on $U(N) / T^{N}$ at the price that we have to take into consideration also spaces homotopy equivalent to $U(N) / T^{N}$. In the following we restrict to the case $G=S^{1}$. We can assume that $\alpha$ maps into aut ${ }_{0}(X)$, the 1 -connected component of aut $(X)$. The graded homomorphisms $H^{*}\left(\operatorname{Baut}_{0}(X) ; \mathbb{Q}\right) \rightarrow$ $H^{*}\left(B_{G} ; \mathbb{Q}\right)$ can be identified with the set of $\mathbb{Q}$-valued points of $V=$ Spec $H^{*}\left(\operatorname{Baut}_{0}(X) ; \mathbb{Q}\right)$. Among all these points we distinguish the subclass of the so called $\mathbf{g}$-points by a purely algebraic condition, see Defs. 4 and 5 . Denote by $G_{m}$ the multiplicative group of $\mathbb{Q}$, i.e., $G_{m} \cong \mathbb{Q}^{*}$. Then up to minor modifications it is shown that the set of $G_{m}$-orbits of g-points classifies $G$-actions on finite $C W$-complexes $Y$ with $H^{*}(Y ; \mathbb{Q}) \cong H^{*}(X ; \mathbb{Q})$ up to equivariant rational homotopy equivalence, see Th. 5. Here the actions $(X, \phi)$ and $(Y, \psi)$ are called rationally homotopy equivalent if there exist a series of intertwining $G$-spaces

$$
(X, \phi) \rightarrow\left(X_{1}, \phi_{1}\right) \leftarrow \cdots \leftarrow\left(X_{i}, \phi_{i}\right) \rightarrow \cdots \leftarrow(Y, \psi)
$$

and equivariant maps inducing isomorphisms in rational homotopy.

As a first application of the theory we consider circle actions on spaces of the rational homotopy type of $\mathbb{C P}^{n}$. As it is well-known, representations of the circle group in a complex vector space $V$ of dimension $n$ are classified by $n$-tuples of weights or more concretely by the $n \times n$ diagonal matrices $\operatorname{diag}\left\{\exp \left(2 \pi \sqrt{-1} a_{i}\right)\right\}$, where the $a_{i}$ are integers. Such a representation induces in a natural way an action on complex projective space, called a linear action of $G$ on projective space. One might ask when two such linear actions are equivariantly $\mathbb{Q}$-homotopy equivalent. Suppose we are given two linear actions $(P V, \rho), \rho=\operatorname{diag}\left\{\exp \left(2 \pi \sqrt{-1} a_{i}\right)\right\}$ and $(P V, \tau), \tau=$ $\operatorname{diag}\left\{\exp \left(2 \pi \sqrt{-1} b_{j}\right)\right\}$ on the complex projective space $P V=\mathbb{C P}^{n}$. Let $\mathcal{S}=T \ltimes E \times \mathcal{S}_{n+1}$ the group generated by the diagonal translations $T$ in the affine space $\mathbb{Q}^{n+1}$, the homotheties $E$ and the symmetric group $\mathcal{S}_{n+1}$ acting 
by permutation of coordinates. Then it is shown in this note, that the representations $\rho$ and $\tau$ are equivariantly $\mathbb{Q}$-homotopy equivalent if and only if there exists a $\Sigma \in \mathcal{S}$ such that $\left(a_{1}, \ldots, a_{n}\right)=\left(b_{1}, \ldots, b_{n}\right)^{\Sigma}$. (Thm. 6 (ii), Thm. 7.) The corresponding quotient space is a moduli space for the equivariant rational homotopy types either of linear actions on $\mathbb{C P}^{n}$ or of arbitrary actions on spaces $X$ with the rational homotopy type of $\mathbb{C P}^{n}$ and can be identified with the quotient of the fundamental Weyl chamber of $S U(n+1)$ modulo the canonical $\mathbb{Q}^{*}$-action

As a second example we consider circle actions on spaces rationally homotopy equivalent to $X=U(N) / T^{N}$. The Weyl group $S_{N}$ of $U(N)$ has a natural action on $V=\operatorname{Spec} H^{*}\left(\operatorname{Baut}_{0}(X) ; \mathbb{Q}\right)$. Then the rational homotopy classes of linear actions on spaces homotopy equivalent to $X$ are classified by the $G_{m}$-orbits of $\mathbf{g}$-points which are in the fixed set $F=F\left(S_{N}, V\right)$. So, an action of $G$ on $U(N) / T^{N}$ is not rationally homotopy equivalent to a linear action iff its $\mathbf{g}$-point lies outside of $F$. It follows from a result of $[\mathbf{9}]$ that there are indeed $\mathbf{g}$-deformations (Def. 4) of the ring $H^{*}\left(U(3) / T^{3}, \mathbb{Q}\right.$ ) which do not correspond to the equivariant cohomology of a linear action on $U(3) / T^{3}$. As it has been shown in $[\mathbf{1 3}]$ these deformations can be realized by circle actions on finite $\mathrm{CW}$-complexes thus showing nonvoidness of the theory. I would like to thank the ancient SFB 170, "Geometrie und Analysis" at Göttingen University for support and the referee for his careful reading of the manuscript and his suggestions.

\section{Equivariant cohomology and localization.}

Suppose $X$ is a topological space and $G$ a topological group which acts continuously on $X$. In transformation group theory one often is led to consider the Borel fibration

$$
X \rightarrow E_{G} \times_{G} X \rightarrow B_{G},
$$

associated to the universal $G$-principal fibration $E_{G} \rightarrow B_{G}$. One puts $X_{G}=$ $E_{G} \times_{G} X$ and calls the ring $H_{G}^{*}(X ; \mathbb{Q})=H^{*}\left(X_{G} ; \mathbb{Q}\right)$ the (ordinary) equivariant rational cohomology. In the following we use always rational coefficients which will be omitted in the text. If we write $R_{G}=H^{*}\left(B_{G}\right)$ the ring $H_{G}^{*}(X)$ has in a natural way the structure of a graded $R_{G}$ algebra. This gives a contravariant functor from the category of $G$-spaces and $G$-maps into the category of graded commutative $R_{G}$ algebras and graded $R_{G}$-algebra homomorphisms. In the particular case where $G$ is a compact connected Lie group and $H^{*}(X)$ a graded $\mathbb{Q}$-algebra vanishing in odd degrees the Serre spectral sequence of the corresponding Borel fibration degenerates at the 2-term and thus the ring $H_{G}^{*}(X)$ becomes a free $R_{G}$ module. Moreover as a consequence of the filtration of the Serre spectral sequence one has $H_{G}^{*}(X) \otimes_{R_{G}} \mathbb{Q} \cong H^{*}(X)$. We are here in a typical algebraic situation, which in algebraic geometry is known as deformation, see e.g., [8]. 
Let $S \subset R_{G}$ be a homogeneously generated multiplicatively closed subset. Denote by $X^{S} \subset X$ the closed $G$-invariant subspace which is defined as follows:

For every point $y \in X$ let $\rho_{y}: R_{G} \rightarrow R_{G_{y}}$ be the homomorphism induced by the inclusion $G_{y} \subset G$ of the isotropy group of $y$. Then we define $I_{y}=$ ker $\rho_{y}$ and we put

$$
X^{S}=\left\{y \in X \mid I_{y} \cap S=\emptyset\right\} .
$$

Definition 1. Let $G$ be a compact Lie group and let $X$ be a $G$-space. Then $X$ will be said to have the $\mathbb{Q}$-localization property (or simply the L-property) if for every homogeneously generated multiplicatively closed subset $S \subset R_{G}$ the (equivariant) inclusion $X^{S} \subset X$ induces an isomorphism

$$
S^{-1} H_{G}^{*}(X) \cong S^{-1} H_{G}^{*}\left(X^{S}\right)
$$

of graded $S^{-1} R_{G}$ algebras.

A localization theorem is a theorem which says that under certain conditions on $G, X$ and the $G$-action on $X$ the L-property is satisfied, for example:

Theorem 1 (Localization theorem of Borel-Quillen-Hsiang). If $G$ is a compact Lie group and if $G$ acts with finitely many orbit types on a compact or paracompact space $X$ which in the case of paracompactness has finite $\mathbb{Q}$-cohomology dimension, then the L-property holds.

As an example, we consider the special case $S=R_{G}-\{0\}$. Then $S^{-1} R_{G}$ is the quotient field of $R_{G}$. We denote by $X^{S}$ the subset of those points $y \in X$ with $I_{y}=(0)$, i.e., the set of all points $y \in X$ where the map $\rho_{y}$ is a monomorphism. Since $\rho_{y}$ is injective if and only if the isotropy group $G_{y} \subset G$ contains a maximal torus $T_{y}$ of $G$, we have $X^{S}=X^{(T)}$ where $X^{(T)}$ is the subset $X^{(T)}=\left\{y \in X \mid r k G_{y}=r k G\right\}$. Therefore we obtain the following isomorphism of $S^{-1} R_{G}$-algebras:

$$
S^{-1} H_{G}^{*}(X) \cong S^{-1} H_{G}^{*}\left(X^{(T)}\right) .
$$

In the case where $G$ is a torus, the set $X^{(T)}$ coincides with the fixed set $X^{G}$ and so we obtain

$$
S^{-1} H_{G}^{*}(X) \cong S^{-1} R_{G} \otimes_{\mathbb{Q}} H^{*}\left(X^{G}\right),
$$

as follows from the obvious isomorphism $H_{G}^{*}(X) \cong R_{G} \otimes_{\mathbb{Q}} H^{*}\left(X^{G}\right)$. We observe that in the finite dimensional case, i.e., if $\operatorname{dim} H^{*}(X)<\infty$, all the $R_{G}$ modules involved are finitely generated and therefore it suffices to invert a single element $\chi_{G}$ for to obtain an isomorphism

$$
H_{G}^{*}(X)\left[\chi_{G}^{-1}\right] \cong H_{G}^{*}\left(X^{G}\right)\left[\chi_{G}^{-1}\right] .
$$


In the case $G=S^{1}$ the cohomology class $\chi_{G}$ can be chosen as the first Chern class of the universal principal bundle $E_{G} \rightarrow B_{G}$ and is therefore a multiplicative generator of the $\mathbb{Q}$-algebra $R_{G}=\mathbb{Q}[t]$. The following lemma will be crucial.

Lemma 1 ([12]). Let $G=S^{1}$ and $X$ be a $G$-space such that the L-property holds. Denote by $\mathbb{Q}_{\eta}=\mathbb{Q}[t] /(t-\eta)$ the residue field of Spec $R_{G}$ in the closed point $t=\eta, \eta \in \mathbb{Q}^{*}$. Then the inclusion $X^{G} \subset X$ induces a (not necessarily graded) isomorphism of $\mathbb{Q}_{\eta}$-algebras

$$
H_{G}^{*}(X) \otimes_{R_{G}} \mathbb{Q}_{\eta} \cong H^{*}\left(X^{G} ; \mathbb{Q}_{\eta}\right) .
$$

Proof. By the L-property the finitely generated $R_{G}$-module $H_{G}^{o d}\left(X, X^{G}\right)$ is a torsion module. By graduation reasons, the only annihilating prime ideal of $H_{G}^{o d}\left(X, X^{G}\right)$ is given by $P=(t)$ and therefore $H_{G}^{*}(X)\left[t^{-1}\right] \cong H_{G}^{*}\left(X^{G}\right)\left[t^{-1}\right]$. Let $\eta \in \mathbb{Q}^{*}$. Tensoring with $\mathbb{Q}_{\eta}=\mathbb{Q}\left[t, t^{-1}\right] /(t-\eta)$ we get the desired result applying the universal coefficient theorem.

Using the terminology of affine algebraic geometry we can say that $H_{G}^{*}\left(X^{G}\right)$ arises as the fiber algebra of the morphism Spec $H_{G}^{*}(X) \rightarrow$ Spec $R_{G}$ over the closed point corresponding to the maximal ideal $(t-\eta), \eta \neq$ 0 .

\section{On the long exact sequence of the pair $\left(X_{G}, X_{G}^{G}\right)$.}

Here we want to investigate a little bit closer the structure of the $R_{G}$-algebra $H_{G}^{*}(X)$ in the particular case when the odd part of $H^{*}(X)$ vanishes. We suppose that $G$ is a circle and $X$ is a $G$-space such that the $\mathbb{Q}$-localization property holds. Let $X^{G}=\sum_{j} F_{j}$ be the decomposition of the fixed set into its connected components $F_{j}$. Denote by $i_{j}^{*}: H_{G}^{*}(X) \rightarrow H_{G}^{*}\left(F_{j}\right)$ the $R_{G}$ algebra homomorphism induced by the inclusion $F_{j} \subset X$ of the $j$-th component. Correspondingly denote by $i^{*}: H_{G}^{*}(X) \rightarrow H_{G}^{*}\left(X^{G}\right)$ the homomorphism induced by the inclusion of the full fixed set $X^{G}$ into $X$. The following is a special case of W. Y. Hsiangs fundamental fixed point theorem, see e.g. [10], [2] (3.8)

Theorem 2. Let $G=S^{1}$, and suppose $X$ is a $G$ space with $H^{o d}(X)=0$ such that the L-property is satisfied. Then the following statements are true:

(i) The long exact cohomology sequence of the pair $\left(E_{G} \times{ }_{G} X, E_{G} \times_{G} X^{G}\right)$ has the form

$$
0 \rightarrow H_{G}^{*}(X) \rightarrow H_{G}^{*}\left(X^{G}\right) \rightarrow H_{G}^{o d}\left(X, X^{G}\right) \rightarrow 0 .
$$

(ii) If (0) $=\cap_{j} Q_{j}$ is the reduced primary decomposition of the zero ideal in $H_{G}^{*}(X)$ then there is a natural bijection between the set $\left\{Q_{j}\right\}_{j}$ and the set $\pi_{0}\left(X^{G}\right)$ of connected components of $X^{G}$ in such a way that there is 
exists a factorization $i_{j}^{*}=\sigma_{j} \circ \mu_{j}$ where $\mu_{j}$ is the canonical projection

$$
\mu_{j}: H_{G}^{*}(X) \rightarrow H_{G}^{*}(X) / Q_{j}
$$

and

$$
\sigma_{j}: H_{G}^{*}(X) / Q_{j} \rightarrow H_{G}^{*}\left(F_{j}\right)
$$

is an injective homomorphism of graded $R_{G}$ algebras such that $C_{j}=$ coker $\sigma_{j}$ is a finitely generated $R_{G}$ torsion module.

(iii) If $P_{j}$ is the radical ideal of $Q_{j}$, i.e., if $P_{j}=\sqrt{Q}_{j}$ then

$$
H_{G}^{*}(X) / P_{j} \cong R_{G} \text {. }
$$

(iv) If $\mathbb{Q}_{\eta}$ is the residue field of $R_{G}$ in the closed point $t=\eta, \eta \neq 0$, then the homomorphism $\sigma_{j}$ induces an ungraded isomorphism

$$
\left(H_{G}^{*}(X) / Q_{j}\right) \otimes_{R_{G}} \mathbb{Q}_{\eta} \cong H^{*}\left(F_{j} ; \mathbb{Q}_{\eta}\right) .
$$

Proof. (i) The long exact sequence of the pair $\left(X, X^{G}\right)_{G}$ can be brought in the form of a hexagon.

$$
\begin{aligned}
& H_{G}^{e v}(X) \longrightarrow H_{G}^{e v}\left(X^{G}\right) \\
& \nearrow \partial_{*} \\
& H_{G}^{e v}\left(X, X^{G}\right) \quad H_{G}^{o d}\left(X, X^{G}\right) . \\
& \partial_{*} \nwarrow \\
& H_{G}^{o d}\left(X^{G}\right) \longleftarrow H_{G}^{o d}(X)
\end{aligned}
$$

From the localization theorem it follows that $H_{G}^{e v}\left(X, X^{G}\right)$ and $H_{G}^{o d}\left(X, X^{G}\right)$ are finite $R_{G}$-torsion modules. Moreover we have $H_{G}^{o d}(X)=0$. It is easy to see that $H_{G}^{o d}\left(X^{G}\right)$ is a free $R_{G^{-}}$module generated by the odd part of $H^{*}\left(X^{G}\right)$. Thus it follows $H_{G}^{e v}\left(X, X^{G}\right)=0$ because it is a torsion submodule of a free module. Consequently $H_{G}^{*}(X) \cong H_{G}^{e v}(X)$ and $H_{G}^{o d}\left(X^{G}\right)=0$.

(ii) It suffices to show that for every connected component $F_{j}$ of $X^{G}$ there exists a primary component $Q_{j}$ such that $Q_{j} \subset$ ker $i_{j}^{*}$. The element $t \in R_{G}$ is a nonzero divisor of $H_{G}^{*}(X)$. Therefore it follows that the primary decomposition of the zero ideal $(0) \subset H_{G}^{*}(X)\left[t^{-1}\right]$ is given by $(0)=\cap_{j=1}^{\rho} Q_{j}\left[t^{-1}\right]$. The localization isomorphism is then given by the canonical homomorphism

$$
H_{G}^{*}(X)\left[t^{-1}\right] \rightarrow \prod_{j=1}^{\rho}\left(H_{G}^{*}(X) / Q_{j}\right)\left[t^{-1}\right]
$$

where

$$
\left(H_{G}^{*}(X) / Q_{j}\right)\left[t^{-1}\right] \cong H_{G}^{*}(F)\left[t^{-1}\right]
$$


Thus it follows that the localized homomorphism $i_{j}^{*}\left[t^{-1}\right]$ vanishes on the localized ideal $Q_{j}\left[t^{-1}\right]$. Therefore $i_{j}^{*}$ vanishes on $Q_{j}$ because $t$ is a nonzero divisor of $Q_{j} \subset H_{G}^{*}(X)$. This implies $Q_{j} \subset \operatorname{ker} i_{j}^{*}$ and therefore we have a factorization $i_{j}^{*}=\sigma_{j} \circ \mu_{j}$ where $\mu_{j}$ is the canonical projection $H_{G}^{*}(X) \rightarrow$ $H_{G}^{*}(X) / Q_{j}$. Now $\sigma_{j}$ induces an isomorphism after inverting $t$. It follows that ker $\sigma_{j}$ and coker $\sigma_{j}$ are $R_{G}$-torsion modules. Because $R_{G}$ is a principal ideal domain the module $H_{G}^{*}(X) / Q_{j}$ is free since it is torsionfree. Moreover it is finitely generated because $H_{G}^{*}(X)$ is finitely generated. It follows that ker $\sigma_{j}$ is finitely generated and therefore we have ker $\sigma_{j}=0$. The proof of (ii) is concluded with the simple observation that $\operatorname{coker} \sigma_{j}$ is also finitely generated.

(iii) We observe that the radical $\sqrt{(0)}$ of $(0)$ in $H_{G}^{*}(F)\left[t^{-1}\right] \cong R_{G}\left[t^{-1}\right] \otimes_{\mathbb{Q}}$ $H^{*}\left(F_{j}\right)$ is generated by the augmentation ideal $H_{+}^{*}\left(F_{j}\right) \subset H^{*}\left(F_{j}\right)$. Thus it follows

$$
\left(H_{G}^{*}(X) / P_{j}\right)\left[t^{-1}\right] \cong R_{G}\left[t^{-1}\right] .
$$

Because $H_{G}^{*}(X)$ is a free graded $R_{G}$ module this implies

$$
H_{G}^{*}(X) / P_{j} \cong R_{G} .
$$

(iv) The statement follows from the fact that $\operatorname{coker} \sigma_{j}$ is a finite torsion module.

The following special case will be of interest in the following.

Lemma 2. If $H^{*}(X)$ is generated as a graded $\mathbb{Q}$-algebra by finitely many elements of degree two, then $\sigma_{j}$ is an isomorphism for all $j$.

Proof. The homomorphism

$$
\sigma_{j}: H_{G}^{*}(X) / Q_{j} \rightarrow R_{G} \otimes_{\mathbb{Q}} H^{*}\left(F_{j}\right)
$$

becomes an isomorphism after inverting the element $t \in R_{G}$. By the assumption and Lemma 1 the $R_{G^{-}}$algebra $H_{G}^{*}(X)$ has generators $X_{1}, \ldots, X_{n}$ which have also degree two. Let $\bar{X}_{i} \in H_{G}^{*}(X) / Q_{j}$ be the residue class of $X_{i}$. It follows that $\sigma_{j}$ has the form

$$
\sigma_{j}\left(\bar{X}_{i}\right)=\omega_{i} \otimes 1+1 \otimes Y_{i}, \omega_{i} \in H^{2}\left(B_{G}\right), Y_{i} \in H^{2}\left(F_{j}\right) .
$$

Since $\sigma_{j}\left[t^{-1}\right]$ is an isomorphism the elements $\sigma_{j}\left(\bar{X}_{i}\right), i=1, \ldots, n$, form a generator system of the $\mathbb{Q}$-algebra $H^{*}\left(F_{j}\right)$. Thus it follows that $\sigma_{j}$ induces an isomorphism

$$
\sigma_{j}: H_{G}^{*}(X) / Q_{j} \otimes_{R_{G}} \mathbb{Q} \stackrel{\cong}{\rightrightarrows} H^{*}\left(F_{j}\right)
$$

of graded $\mathbb{Q}$-algebras. In particular one has coker $\sigma_{j} \otimes_{R_{G}} \mathbb{Q}=0$. By the Lemma of Nakayama this implies coker $\sigma_{j}=0$ and therefore the result follows from the preceding theorem. 
Thus in particular the long exact sequence of the pair $\left(X_{G}, X_{G}^{G}\right)$ degenerates into the canonical exact sequence

$$
0 \rightarrow H_{G}^{*}(X) \rightarrow \prod_{j=1}^{\rho} H_{G}^{*}(X) / Q_{j} \rightarrow \operatorname{coker} \mu \rightarrow 0 .
$$

This motivates the following definition.

Definition 2. Let $k$ be a field, let $R=k[t], \operatorname{deg} t=2$, and let $A$ be a graded $R$-algebra which is free as an $R$-module. Then an exact sequence $0 \rightarrow A \rightarrow B \rightarrow Q \rightarrow 0$ will be called a g-sequence if:

(i) There exists a family $B_{j}, j=1, \ldots \rho$, of graded artinian $k$-algebras such that $B \cong \prod_{j} R \otimes_{k} B_{j}$.

(ii) The cokernel $Q$ is a finite $R$-torsion module.

In [13] it has been shown that any g-sequence is given by the equivariant cohomology ring of a $G$-action on some finite $G$-CW-complex. Conversely we will show that under some circumstances the rational homotopy type (and even more) of a circle action is determined by its $\mathbf{g}$-sequence given by equivariant cohomology.

\section{F-spaces and formality of maps.}

As defined in [6] an F-space is a 1-connected topological space with finite dimensional rational cohomology and finite dimensional rational homotopy. If $X$ is an F-space with $H^{o d}(X)=0$, then it is shown in [6] that $H^{*}(X)$ is a quasihomogeneous complete intersection of finite length. Such a space is formal in the sense of rational homotopy theory, i.e., its minimal model $\mathcal{M}(X)$ is a formal consequence of its rational cohomology. Consequently there exists a D.G.A.-morphism $\rho_{X}: \mathcal{M}(X) \rightarrow H^{*}(X)$ inducing an isomorphism in cohomology considering $H^{*}(X)$ as a differential graded algebra (D.G.A.) with $d=0$.

In the following let $k$, char $k=0$, be a fixed field, let $A_{0} \mid k$ be a complete intersection with positive graduation:

$$
A_{0}=k\left[x_{1}, \ldots, x_{n}\right] / I_{0},
$$

where the generators $x_{i}$ have weights $\left|x_{i}\right|=d_{i} \equiv 0(\bmod 2)$. Then the ideal $I_{0}$ is generated by a prime series $\left\{f_{1}, \ldots, f_{m}\right\}, m \leq n$, of quasi-homogeneous elements. We consider $A_{0}$ as D.G.A. with $d=d_{A_{0}}=0$. Let $\left(\mathcal{M}\left(A_{0}\right), d\right)$ be the corresponding minimal model. Then it can be shown that $\left(\mathcal{M}\left(A_{0}\right), d\right)$ is isomorphic in the D.G.A.-sense to

$$
\mathcal{M}\left(A_{0}\right)=k\left[\xi_{1}, \ldots, \xi_{n}\right] \otimes_{k} \Lambda\left(\beta_{1}, \ldots, \beta_{m}\right),
$$

where the degrees are given by

$$
\left|\xi_{i}\right|=d_{i}
$$




$$
\left|\beta_{j}\right|=\left|f_{j}\right|-1
$$

and the differential is

$$
\begin{gathered}
d \xi_{i}=0, i=1, \ldots, n, \\
d \beta_{j}=f_{j}\left(\xi_{1}, \ldots, \xi_{n}\right), j=1, \ldots, m .
\end{gathered}
$$

Moreover, a formality map $\rho: \mathcal{M}\left(A_{0}\right) \rightarrow A_{0}$ is given by

$$
\begin{gathered}
\rho\left(\xi_{i}\right)=\bar{x}_{i}=x_{i}+I_{0}, \\
\rho\left(\beta_{j}\right)=0 \in A_{0} .
\end{gathered}
$$

Consequently, $\rho$ can be identified with the natural projection map

$$
k[(\xi)] \otimes_{k} \Lambda((\beta)) \rightarrow k[(\xi)] \otimes_{k} \Lambda((\beta)) / I_{0}^{\prime},
$$

where $I_{0}^{\prime}$ is the D.G.A.-ideal generated by $I_{0}$ and the elements $\beta_{j}$. For these and similar observations see $[\mathbf{2}, \mathbf{3}, \mathbf{6}]$. We recall that the above considerations do not depend on the number of generators of the ideal $I_{0}$, i.e., the complete intersections need not be artinian. In the following we fix once for all this kind of formality map. The following is a simple but useful observation. The proof will be left to the reader. As usual, an algebra $A$ with a unique maximal ideal $\underline{m}_{A}$ will be called local. In our case the maximal ideal of $A$ is identical with the augmentation ideal $A_{+}$.

Lemma 3. Let $A$ and $B$ local graded complete intersections considered as D.G.A.s with trivial differential. Let $\mathcal{M}(A)$ and $\mathcal{M}(B)$ be the corresponding minimal models and let $I$ and $J$, respectively, be ideals with $A=\mathcal{M}(A) / I$ and $B=\mathcal{M}(B) / J$. Let $F: \mathcal{M}(A) \rightarrow \mathcal{M}(B)$ a D.G.A.-homomorphism of the corresponding minimal models. Then the map $H(F)$ induced in homology coincides with the canonical map induced by $F$ on the respective quotients.

Therefore, if we fix the formality maps $\rho_{A}$ and $\rho_{B}$ in the way indicated above, the diagram

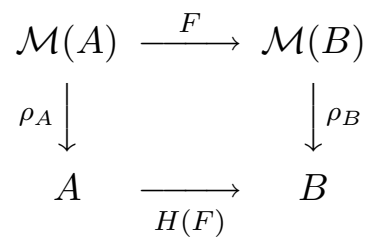

will be commutative.

Let $A=\prod_{i} A_{i}$ and $B=\prod_{j} B_{j}$ be graded $k$-algebras, let $f: A \rightarrow B$ be a graded $k$-algebra homomorphism, then one can write $f$ as a matrix $\left(f_{i j}\right)$ of graded ring homomorphisms $f_{i j}: A_{i} \rightarrow B_{j}$ of connected components. It follows from the multiplicativity of $f$ that for any $B_{j}$ there is at most one $A_{i}$ with $f_{i j} \neq 0$. 
Lemma 4. Let $A=\prod_{i} A_{i}$ and $B=\prod_{j} B_{j}$, where the local components $A_{i}$ and $B_{j}$ are graded complete intersections. Let $f: A \rightarrow B$ be a graded homomorphism of graded $k$-algebras. Then there exists a D.G.A.-morphism $F: \mathcal{M}(A) \rightarrow \mathcal{M}(B)$ such that the diagram

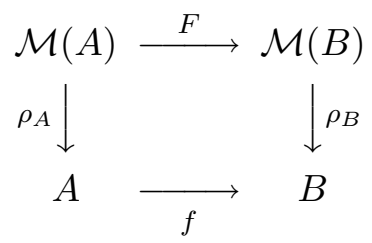

is commutative and such that $f$ coincides with the homomorphism $H(F)$ induced in homology by $F$.

Proof. The minimal models $\mathcal{M}(A)$ and $\mathcal{M}(B)$ are products

$$
\begin{aligned}
& \mathcal{M}(A)=\prod_{i} \mathcal{M}\left(A_{i}\right), \\
& \mathcal{M}(B)=\prod_{j} \mathcal{M}\left(B_{j}\right) .
\end{aligned}
$$

The homomorphism $f$ is a matrix $\left(f_{i j}\right)$ of graduation preserving homomorphisms $f_{i j}: A_{i} \rightarrow B_{j}$ of complete intersections. First we have to show that there is a lifting $F: \mathcal{M}(A) \rightarrow \mathcal{M}(B)$ of $f: A \rightarrow B$ when $A$ and $B$ are connected.

There are quite general theorems which can be applied to this situation, for example (2.2.9) Theorem in [2]. By the simplicity of the situation it seems reasonable to give an explicit lifting using the above observations on minimal models of complete intersections.

Let $A=P / I$ where $P=k\left[x_{1}, \ldots, x_{n}\right]$ is a homogeneous polynomial algebra and $I \subset\left(x_{1}, \ldots, x_{n}\right)$ an ideal generated by a maximal length prime series $f_{1}, \ldots, f_{n}$ of homogeneous elements. Analogously write $B=Q / J$ with $Q=k\left[y_{1}, \ldots, y_{m}\right]$ and $J=\left(g_{1}, \ldots, g_{m}\right)$. Then choose a graded homomorphism $\tilde{F}: P \rightarrow Q$ inducing $f$ on the quotients. Let $\tilde{F}\left(x_{i}\right)=p_{i}\left(y_{1}, \ldots, y_{m}\right)$. Since $\tilde{F}(I) \subset J$, one can choose a matrix $\left(\lambda_{i j}\right)$ of homogeneous elements $\lambda_{i j} \in Q$ such that $\tilde{F}\left(f_{i}\right)=\sum_{j} \lambda_{i j} g_{j}$ in $Q$. Write the minimal model of $\left(\mathcal{M}(A), d_{A}\right)$ of $(A, d=0)$ as

$$
\mathcal{M}(A)=k\left[\xi_{1}, \ldots, \xi_{n}\right] \otimes \Lambda\left(\beta_{1}, \ldots, \beta_{n}\right)
$$

with $d_{A} \xi_{i}=0$ and $d_{A} \beta_{j}=f_{j}\left(\xi_{1}, \ldots, \xi_{n}\right)$ and formality map $\rho_{A}\left(\xi_{i}\right)=$ $\bar{x}_{i}, \rho_{A}\left(\beta_{j}\right)=0$. Similarly write the minimal model $\left(\mathcal{M}(B), d_{B}\right)$ of $(B, d=0)$ as

$$
\mathcal{M}(B)=k\left[\eta_{1}, \ldots, \eta_{m}\right] \otimes \Lambda\left(\gamma_{1}, \ldots, \gamma_{m}\right)
$$


with $d_{B} \eta_{i}=0$ and $d_{B} \gamma_{j}=g_{j}\left(\eta_{1}, \ldots, \eta_{m}\right)$ where the formality map is given by $\rho_{B}\left(\eta_{i}\right)=\bar{y}_{i}$ and $\rho_{B}\left(\gamma_{j}\right)=0$. Then define $F: \mathcal{M}(A) \rightarrow \mathcal{M}(B)$ by

$$
F\left(\xi_{i}\right)=p_{i}\left(\eta_{1}, \ldots, \eta_{m}\right), \quad i=1, \ldots n
$$

and

$$
F\left(\beta_{j}\right)=\sum_{k} \lambda_{j k}\left(\eta_{1}, \ldots, \eta_{m}\right) \gamma_{k}, \quad j=1, \ldots n .
$$

One simply verifies that $F$ is welldefined. Moreover, one has $d_{B} \circ F=F \circ d_{B}$, i.e., $F$ defines a D.G.A.-morphism. By the previous lemma one has $H(F)=$ $f$ since by construction $F$ induces $f$ on the quotients. It follows that for all $(i, j)$ with $f_{i j} \neq 0$ we have a D.G.A.-morphism $F_{i j}$ and a commutative diagram

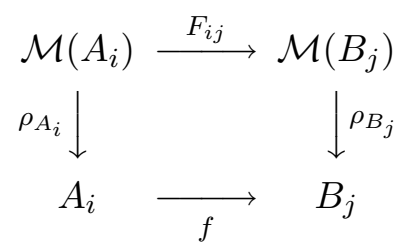

with $H\left(F_{i j}\right)=f_{i j}$. Then define $F$ as the matrix $F=\left(\Phi_{i j}\right)$ where $\Phi_{i j}=F_{i j}$, if $f_{i j} \neq 0$ and $\Phi_{i j}=0$ if $f_{i j}=0$. Thus it follows that for any $j$ there is at most one $i$ with $F_{i j} \neq 0$. For to show multiplicativity of $F$ it suffices therefore to consider the case when $A$ is connected. We therefore can assume that $F$ has the form of a column matrix $F=\left(F_{j}\right)$, where for any $j$ the map $F_{j}: \mathcal{M}(A) \rightarrow \mathcal{M}\left(B_{j}\right)$ is multiplicative. But since $\mathcal{M}(B)=\prod_{j} \mathcal{M}\left(B_{j}\right)$ is the direct product, $F$ is multiplicative if and only if $F_{j}$ is multiplicative for any $j$.

Recall that the cohomology ring with rational coefficients of the classifying space $B_{G}$ of a compact Lie group $G$ is a graded commutative polynomial algebra in finitely many even generators. It follows that the minimal model $\mathcal{M}\left(B_{G}\right)$ of $B_{G}$ is isomorphic to $R_{G}=H^{*}\left(B_{G}\right)$ considered as a differential graded algebra with trivial differential. Suppose $G$ is acting on a 1-connected $F$-space $X$ with vanishing odd rational cohomology. Then the $\mathbb{Q}$-algebras $H^{*}(X)$ and $H_{G}^{*}(X)$ are complete intersections. Moreover $H_{G}^{*}(X)$ is a $R_{G^{-}}$ relative complete intersection and the minimal model $\mathcal{M}\left(X_{G}\right)$ becomes a $R_{G}$-algebra such that the formality map $\rho_{G}: \mathcal{M}\left(X_{G}\right) \rightarrow H_{G}^{*}(X)$ is also a $R_{G}$-module homomorphism.

Definition 3. Let $G$ be a torus and suppose we are given two $G$-actions on 1-connected spaces $X$ and $Y$ respectively. Let $H_{G}^{*}(X)$ and $H_{G}^{*}(Y)$ be free $R_{G}$-modules. Then the two $G$-spaces are called similar if there exists 
a commutative diagram of $R_{G^{-}}$-algebra homomorphisms

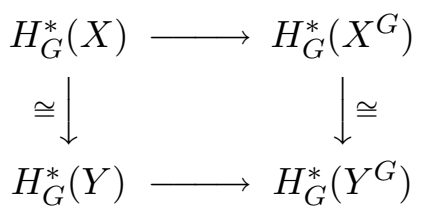

where the vertical maps are isomorphisms and the horizontal maps are induced by the respective inclusions of the fixed sets.

Of course, the simple existence of an $R_{G}$-algebra homomorphism $H_{G}^{*}(X)$ $\cong H_{G}^{*}(Y)$ does not imply similarity. Nonetheless this is true under certain special hypotheses, for example, if $H^{*}(X)$ is generated as $\mathbb{Q}$-algebra by elements of degree two. It is clear that similarity is an equivalence relation between $G$ - actions.

Theorem 3. Suppose $X$ and $Y$ to be F-spaces without odd cohomology. Let a torus $G$ act on both spaces with the respective fixed spaces $X^{G}$ and $Y^{G}$. Suppose there is a commutative diagram

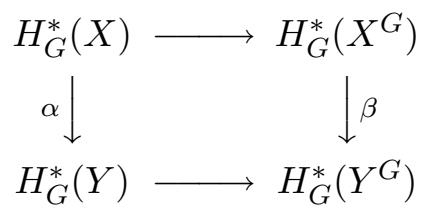

of $R_{G^{-}}$-algebra homomorphisms where the horizontal maps are induced by the respective inclusions. Then there exists a homotopy commutative diagram

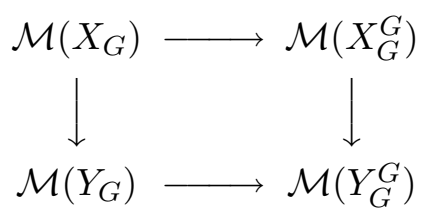

of $R_{G}$ - D.G.A.-maps of the minimal models inducing the diagram (3) in (co)homology.

Proof. By $[6]$ the rings $H^{*}(X)$ and $H^{*}(Y)$ are complete intersections. Then by standard results in cohomology theory of transformation groups the local components of $H^{*}\left(X^{G}\right)$ and $H^{*}\left(Y^{G}\right)$ are complete intersections too, see e.g., [5] or for example the result of Allday, [1] on the homotopy Euler characteristic of a fixed space component, see also 3.3.11 and (3.8.12) Theorem in [2]. Then the statement is an immediate consequence of Lemma 4.

\section{Similarity implies equivariant rational homotopy equivalence.}

Let $G$ be a compact Lie group acting on the spaces $X$ and $Y$. Then the two actions are called weakly equivariantly rationally homotopy equivalent if there is a finite chain $X_{0}, X_{1}, \ldots, X_{n}$ of $G$-spaces with $X_{0}=X$ and $X_{n}=$ 
$Y$ such that for all $i, i=0, \ldots, n$, there exists either an equivariant map $f_{i}: X_{i} \rightarrow X_{i+1}$ or an equivariant map $f_{i}: X_{i} \rightarrow X_{i-1}$ which are inducing rational homotopy equivalences. Therefore we have something like

$$
X_{0} \rightarrow X_{1} \leftarrow X_{2} \leftarrow \cdots \rightarrow X_{n} .
$$

This gives an equivalence relation on the set of $G$-spaces.

Remarks 1. The spaces $X=X_{0}$ and $Y=X_{n}$ are $\mathbb{Q}$-homotopy equivalent if and only if we are in one of the following four cases:

$$
\begin{aligned}
X_{0} & \leftarrow X_{1} \rightarrow X_{2} \\
X_{0} & \rightarrow X_{1} \\
X_{0} & \leftarrow X_{1} \\
X_{0} & =X_{1}
\end{aligned}
$$

as can be shown in the following way: We can assume that successive arrows have always opposite direction otherwise we take the composition. Suppose now that the length of the diagram is bigger than four. Then it contains a subdiagram either of type

or

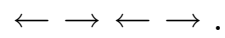

But by successively forming pullbacks one can reduce the first type to

The second type can be reduced by forming the pullback of $\rightarrow \leftarrow$ and then composing. So in both cases we arrive at the type $\longleftarrow \longrightarrow$. This gives a modulo two reduction of all diagrams of length bigger than four and thus proves the observation.

If $X=X_{0}$ and $Y=X_{n}$ are finite $G$-CW complexes one also can take finite $G$-CW complexes for the intertwining spaces $X_{1}, \ldots, X_{n-1}$. The equivalence class of a $G$-space will be called its weak equivariant rational homotopy type. Let $X \leftarrow Z \rightarrow Y$ be a weak equivariant rational homotopy equivalence. Then, since the maps are equivariant, for every subgroup $H \subset G$ there is an induced diagram

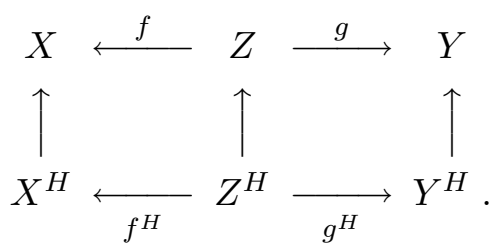

If $\alpha=\left(g^{*}\right)^{-1} \circ f^{*}$ and $\beta=\left(\left(g^{H}\right)^{*}\right)^{-1} \circ\left(f^{H}\right)^{*}$ it follows that a weak equivariant rational homotopy equivalence for every closed subgroup $H \subset G$ induces a 
commutative diagram

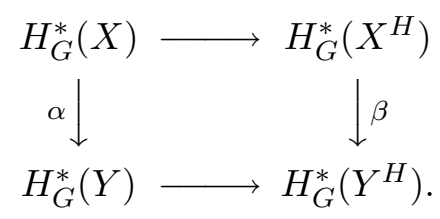

Here the first vertical homomorphism is an isomorphism whereas the second in general is not. It can however be an isomorphism under special conditions on the graduation of $H^{*}(X)$ and $H^{*}\left(X^{G}\right)$, for example in the case of a torus action $\beta$ is also an isomorphism if $H^{*}(X)$ is multiplicatively generated by elements of degree two.

On the other hand one can define strong equivariant rational homotopy equivalence between $G$-spaces $X_{0}$ and $X_{n}$ as a sequence of commutative diagrams of rational homotopy equivalences

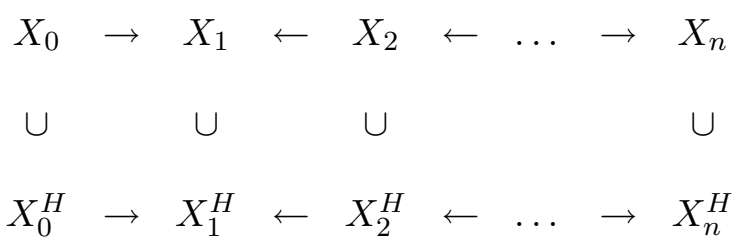

for any closed subgroup $H \subseteq G$ where the maps in the upper row are $G$ equivariant and the maps in the lower row are equivariant with respect to the natural $N_{G} H / H$-actions. This again gives an equivalence relation. The equivalence class of a $G$-space will be called its strong rational homotopy type or simply its rational homotopy type. For our purpose we consider a somewhat intermediate category requesting the existence of such a diagram for closed connected subgroups $H$ only. In the following this functor will be called the equivariant connected $\mathbb{Q}$-homotopy type. In the case of $G=$ $S^{1}$ equivariant cohomology gives therefore a functor from the category of equivariant connected $\mathbb{Q}$-homotopy types of $G$-actions on 1 -connected spaces $X$ with $H^{o d}(X)=0$ to the category of similarity classes of $\mathbf{g}$-sequences. In [13] it was shown that the above functor is surjective, i.e., to every exact sequence $\gamma=\{0 \rightarrow A \rightarrow R \otimes \bar{B}\}$ there exists a finite G-CW complex $\left(X, X^{G}\right)$ such that the corresponding exact cohomology sequence of the pair $\left(X, X^{G}\right)_{G}$ is similar to the given exact sequence. In this paragraph we intend to prove the injectiveness of the above functor.

Theorem 4. Let $G=S^{1}$ and let $X$ and $Y$ be 1-connected $G$-spaces with vanishing odd dimensional rational cohomology. Let $H^{*}(X)$ and $H^{*}(Y)$ be complete intersections of finite length and suppose that in both cases the $L$ property is satisfied. Moreover, suppose $X^{G}$ and $Y^{G}$ to be componentwise simply connected. Then similarity of the $G$-actions implies equivariant $\mathbb{Q}$ homotopy equivalence. 
If $X$ and $Y$ are similar $G$-spaces satisfying the above conditions, the proof will consist in the construction of a third $G$-space $Z$ with a commutative diagram

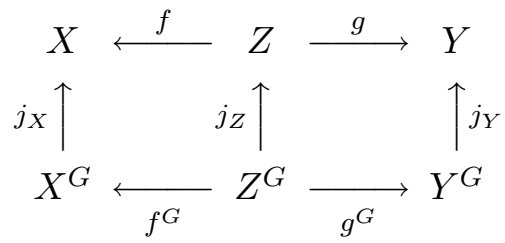

where $f$ and $g$ are equivariant maps which induce rational homotopy equivalences. Here the maps $f^{G}$ and $g^{G}$ induced on the fixed sets are also rational homotopy equivalences. The maps $j_{X}, j_{Y}$ and $j_{Z}$ denote the inclusions of the respective fixed sets.

Proof. We have a commutative diagram

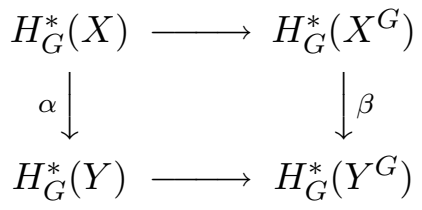

of $R_{G}$-algebras and $R_{G}$-algebra homomorphisms where the vertical maps are isomorphisms. In the first step of the proof we begin with the construction of the fixed set $Z_{0}=Z^{G}$. First recall that every local component of the rings $H^{*}\left(X^{G}\right) \cong H^{*}\left(Y^{G}\right)=B_{0}$ is a graded complete intersection [5]. Therefore the rational homotopy type of $Z^{G}$ is uniquely determined by the isomorphism type of $B_{0}$. In the following we first assume $B_{0}$ to be local, i.e., $X^{G}$ and $Y^{G}$ connected, and try to construct a finite componentwise 1-connected CW-complex $Z_{0}$ with $H^{*}\left(Z_{0}\right)=B_{0}$. This will be done stepwise. In the first step we take a $\mathbb{Q}$-space $\tilde{Z}_{0}$ with $H^{*}\left(\tilde{Z}_{0} ; \mathbb{Z}\right) \cong B_{0}$. The existence of such a space is an immediate consequence of Sullivan theory. Moreover one has maps

$$
X_{\mathbb{Q}}^{G} \stackrel{\tilde{f}_{0}}{\longleftarrow} \quad \tilde{Z}_{0} \stackrel{\tilde{g}_{0}}{\longrightarrow} Y_{\mathbb{Q}}^{G}
$$

into the corresponding localizations inducing homotopy equivalences. This also follows from Sullivan theory. Let now $e=e \operatorname{dim} B_{0}$ be the embedding dimension of this component, i.e., $e$ is the number of multiplicative generators of $B_{0}$ as a graded $\mathbb{Q}$-algebra. Let $d_{1} \leq d_{2} \leq \cdots \leq d_{e}$ be the degrees of the generators of a minimal homogeneous generator system of $B_{0}$ and consider the wedge

$$
Z_{0}^{1}=S^{d_{1}} \vee S^{d_{2}} \vee \cdots \vee S^{d_{e}}
$$


Then we have a commutative diagram

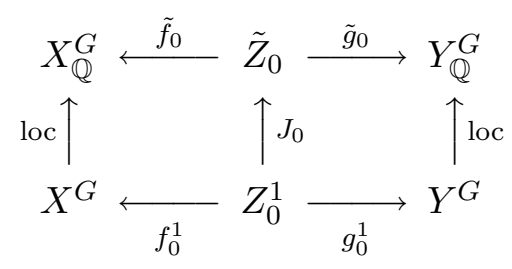

where the left and the right vertical maps are the respective localization maps. Moreover the multiplicative generators of degrees $d_{1}, \ldots, d_{e}$ respectively are spherical (since $X^{G}$ and $Y^{G}$ are componentwise simply connected) and thus correspond to maps $J_{0}^{i}: S^{d_{i}} \rightarrow \tilde{Z}_{0}$ thus inducing $J_{0}=J_{0}^{1} \vee \cdots \vee J_{0}^{e}$. In the same way we obtain the maps $f_{0}^{1}$ and $g_{0}^{1}$. In the next steps by standard algebraic topology we successively attach cells to $Z_{0}^{1}$ and extend the maps. This process must come to an end because $B_{0}$ is a finite $\mathbb{Q}$-vector space. So we have gotten a finite CW-complex $Z_{0}, H^{*}\left(Z_{0}\right) \cong B_{0}$, and a diagram

$$
X^{G} \stackrel{f_{0}}{\longleftarrow} Z_{0} \stackrel{g_{0}}{\longrightarrow} Y^{G}
$$

where $f_{0}, g_{0}$ are rational homotopy equivalences such that $\beta=g_{0}^{*-1} \circ f_{0}^{*}$. The details will be left to the reader.

We come now to the equivariant part of the proof.

Let $A=H_{G}^{*}(X), A_{0}=H^{*}(X)$ and let $R_{G}=H^{*}\left(B_{G}\right)$. We begin with the construction of a $\mathbb{Q}$-fibration

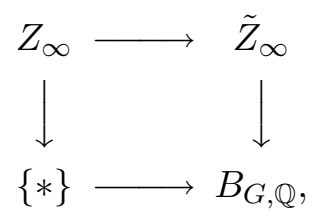

which realizes the commutative diagram

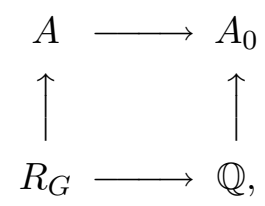

i.e., $H^{*}\left(Z_{\infty} ; \mathbb{Z}\right) \cong A_{0}$ and $H^{*}\left(\tilde{Z}_{\infty} ; \mathbb{Z}\right) \cong A$ with $H^{*}\left(\tilde{Z}_{\infty} ; \mathbb{Z}\right) \otimes_{R_{G}} \mathbb{Q} \cong$ $H^{*}\left(Z_{\infty} ; \mathbb{Z}\right)$. Recall that the existence of such a fibration is an immediate consequence of Sullivan theory, see e.g., [8], no. 3. We insert the injective homomorphism $A \rightarrow H^{*}\left(B_{G} \times Z_{0}\right)$ into the diagram (13) thus obtaining a 
commutative diagram

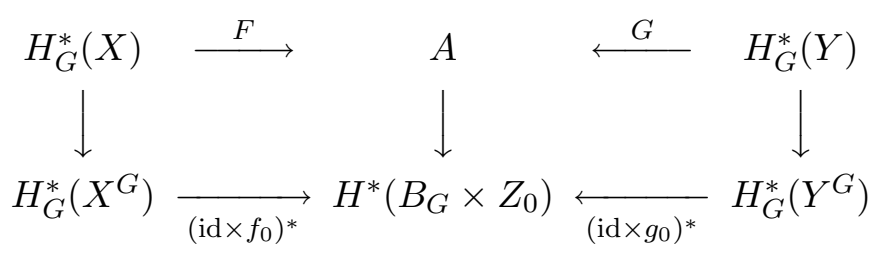

where $F$ and $G$ are isomorphisms with $\alpha=G^{-1} \circ F$. If we apply Lemma 5 to the homomorphisms in diagram (19), we obtain a homotopy commutative diagram

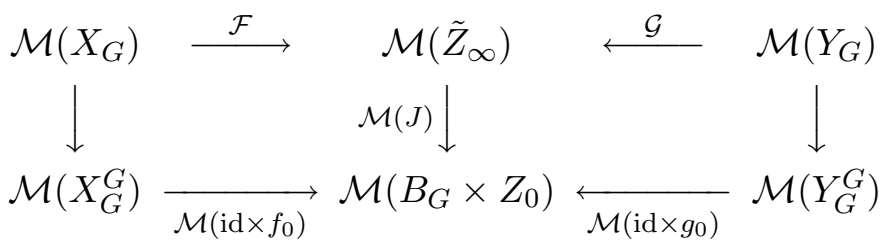

covering diagram (19) in the sense of Theorem 3 . Using the fibration $\tilde{Z}_{\infty} \rightarrow$ $B_{G, \mathbb{Q}}$ as a model we want to realize in a bootstrap technique a Borel fibration by attaching equivariant cells to $Z_{0}$ and simultaneously extend the maps $f_{0}$ and $g_{0}$ to equivariant maps $f_{i}$ and $g_{i}$ on a space $Z_{i}$. Moreover we will extend $J_{0}$ to $J_{i}: E_{G} \times_{G} Z_{i} \rightarrow \tilde{Z}_{\infty}$ until it has become a rational homotopy equivalence.

In the first step of the construction of the $G-C W$-complex $Z$ we make $Z_{0}$ connected by attaching as many equivariant one-cells $G \times e^{1}$ as necessary to kill the first homology group of $J$. This means that we build bridges of $S^{2}$-type between the connected components of $Z_{0}$, where $G$ is acting on such a bridge with two isolated fixed points anchored in two different components of $Z_{0}$. Then it is clear that the map $J_{0}$ can be extended and $f_{0}$ and $g_{0}$ can be extended equivariantly. We may therefore assume that $Z$ is connected and that the mapping cone $C=C_{J}$ of $J$ is one-connected.

Let $Z=Z_{i}$ and let $J=J_{i}, f=f_{i}$ and $g=g_{i}$. Thus we can suppose that we have the following commutative diagram of minimal models.

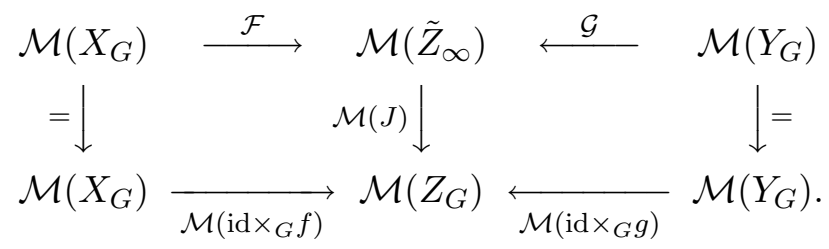

Let $M=M_{J}$ be the mapping cylinder of $J$. Recall that $H_{G}^{o d}(Z)=H^{o d}\left(\tilde{Z}_{\infty} ; \mathbb{Z}\right)$ $=0$. Then there is a minimal odd number $2 m+1 \in \mathbb{N}, m>0$, with the property $r k H^{i}\left(M, Z_{G}\right)=0$ for all $i<2 m+1$ and $H^{2 m+1}\left(M, Z_{G}\right) \neq 0$. Consider the exact cohomology sequence

$$
0 \rightarrow H^{2 m}\left(\tilde{Z}_{\infty}\right) \rightarrow H^{2 m}\left(Z_{G}\right) \rightarrow H^{2 m+1}\left(M, Z_{G}\right) \rightarrow 0 .
$$


Then there is an corresponding exact sequence in homology

$$
0 \rightarrow H_{2 m+1}\left(M, Z_{G}\right) \stackrel{\partial_{*}}{\rightarrow} H_{2 m}\left(Z_{G}\right) \rightarrow H_{2 m}\left(\tilde{Z}_{\infty} ; \mathbb{Z}\right) \rightarrow 0 .
$$

Recall that $Z, Z_{G}$ and $\tilde{Z}_{\infty}$ are 1-connected. Now the rational Hurewicz homomorphism gives a diagram which is commutative up to sign with exact rows where the first vertical arrow is an isomorphism of $\mathbb{Q}$-vector spaces. Here $\pi_{i}^{\mathbb{Q}}(-)$ means $\pi_{i}(-) \otimes_{\mathbb{Z}} \mathbb{Q}$.

$$
\begin{aligned}
& 0 \rightarrow H_{2 m+1}\left(M, Z_{G}\right) \stackrel{\partial}{\longrightarrow} H_{2 m}\left(Z_{G}\right) \longrightarrow H_{2 m}\left(\tilde{Z}_{\infty} ; \mathbb{Z}\right) \rightarrow 0
\end{aligned}
$$

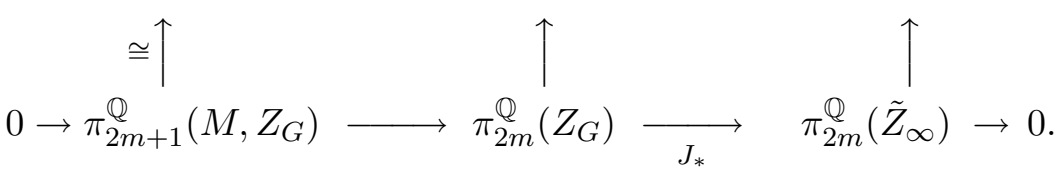

The diagram (21) induces a commutative diagram of rational homotopy groups.

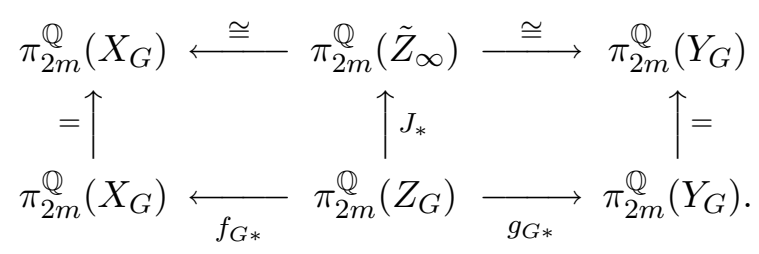

Let $g^{\prime} \in H_{2 m+1}\left(M, Z_{G}\right)$ be a nonzero element, let $c^{\prime} \in \pi_{2 m+1}^{\mathbb{Q}}\left(M, Z_{G}\right)$ be the corresponding element under the Hurewicz isomorphism and let $c=$ $\partial_{*}\left(c^{\prime}\right) \in \pi_{2 m}^{\mathbb{Q}}\left(Z_{G}\right)$. Then we have $J_{*}(c)=0, f_{G *}(c)=0$ and $g_{G *}(c)=0$. Let $\chi_{G}: \partial e^{2 m+1} \rightarrow Z_{G}$ be a map with $\left[\chi_{G}\right]=c$, then we can conclude that $f_{G *}\left[\chi_{G}\right], g_{* G}\left[\chi_{G}\right]$ and $J_{*}\left[\chi_{G}\right]$ are torsion elements. Let $\alpha, \beta$ and $\gamma$ be the minimal nonvanishing natural numbers such that

$$
\begin{gathered}
\alpha f_{G *}\left[\chi_{G}\right]=0, \\
\beta g_{G *}\left[\chi_{G}\right]=0, \\
\gamma J_{*}\left[\chi_{G}\right]=0 .
\end{gathered}
$$

Let $n=$ l.c.m. $(\alpha, \beta, \gamma)$ and substitute $\chi_{G}: \partial e^{2 m+1} \rightarrow Z_{G}$ by its $n$-multiple $n\left[\chi_{G}\right]$. Then it follows that the maps $f_{G} \circ \chi_{G}, g_{G} \circ \chi_{G}$ and $J \circ \chi_{G}$ are nullhomotopic.

Lemma 5. Let $m \geq 1$ be a number as above, let $g^{\prime} \in H_{2 m+1}\left(M, Z_{G}\right)$ and let $c^{\prime} \in \pi_{2 m+1}\left(M, Z_{G}\right)$ be the element corresponding to $g^{\prime}$ under the Hurewicz isomorphism. Let $\left[\chi_{G}\right]=\partial_{*}\left(c^{\prime}\right) \in \pi_{2 m}\left(Z_{G}\right)$. Let $i_{b}: Z \rightarrow Z_{G}$ be the inclusion of the fiber with respect to a base point $b$. Then there is a map $\chi: \partial e^{2 m+1} \rightarrow Z$ with $i_{b} \circ \chi \simeq \chi_{G}$. Moreover $f \circ \chi$ and $g \circ \chi$ are nullhomotopic iff $f_{G} \circ \chi_{G}$ and $g_{G} \circ \chi_{G}$ are.

Proof. $m>1$ : The statement follows from the surjectivity of the map $\pi_{2 m}(Z) \rightarrow \pi_{2 m}\left(Z_{G}\right)$ induced by $i_{b}$. 
$m=1$ : Let $g^{\prime} \in H_{3}\left(M, Z_{G}\right)$ be nonzero, let $c^{\prime} \in \pi_{3}\left(M, Z_{G}\right)$ be the element corresponding to $g^{\prime}$ under the Hurewicz isomormorphism. Since the exact sequence

$$
0 \rightarrow H^{*}\left(\tilde{Z}_{\infty}\right) \rightarrow H^{*}\left(Z_{G}\right) \rightarrow H^{*+1}\left(M, Z_{G}\right) \rightarrow 0
$$

is a sequence of $R_{G}$-modules, we have a commutative diagram

$$
\begin{aligned}
& \pi_{2}(Z) \\
& \begin{array}{rlcc}
\pi_{3}\left(M, Z_{G}\right) \rightarrow & \pi_{2}\left(Z_{G}\right) & \rightarrow & \pi_{2}(\tilde{Z}) \\
\downarrow & & \downarrow \\
\pi_{2}\left(B_{G}\right) & = & \pi_{2}\left(B_{G}\right)
\end{array}
\end{aligned}
$$

where the middle horizontal and the middle vertical sequences are exact. Thus it follows $c=\partial_{*}\left(c^{\prime}\right) \in \operatorname{Im} i_{b *}$. Therefore there are maps $\chi_{G}$ and $\chi$ such that $i_{b} \circ \chi$ and $\chi_{G}$ are homotopic. Moreover the inclusion of the fiber induces an injection in the homotopy groups for the Borel fibrations $X_{G} \rightarrow B_{G}$ and $Y_{G} \rightarrow B_{G}$ which proves the statement.

Let now $\phi: G \times \partial e^{2 m+1} \rightarrow Z$ be the equivariant map $\phi(g, e)=g \chi(e)$. Let $\phi_{G}: E_{G} \times_{G}\left(G \times \partial e^{2 m+1}\right) \rightarrow Z_{G}$ be the induced map. Let $e_{1} \in E_{G}$ be a fixed point which projects down to the point $b \in B_{G}$. Let $j_{1}: 1 \times \partial e^{2 m+1} \rightarrow$ $E_{G} \times \partial e^{2 m+1}$ be the inclusion $j_{1}(1, e)=\left(e_{1}, e\right)$. Then we have a commutative diagram

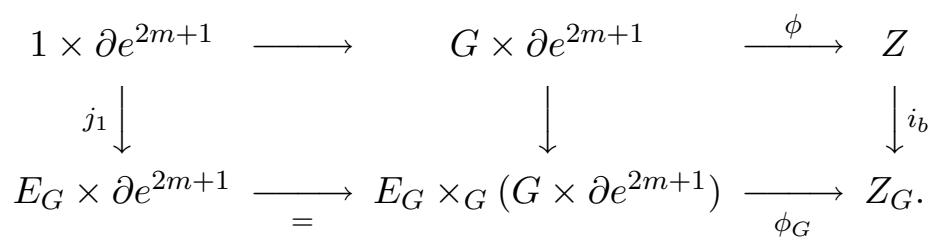

Thus it follows $\phi_{G} \circ j_{1}=i_{b} \circ \phi=i_{b} \circ \chi \simeq \chi_{G}$. So, attaching a free $G$-cell $G \times e^{2 m+1}$ to $Z$ by the equivariant map $\phi: G \times \partial e^{2 m+1} \rightarrow Z, \phi(g, e)=$ $g \cdot \chi(e)$, homotopy-theoretically means to attach a homotopy cell $E_{G} \times{ }_{G}$ $\left(G \times e^{2 m+1}\right) \simeq e^{2 m+1}$ to $Z_{G}$ by the map $\chi_{G}: \partial e^{2 m+1} \rightarrow Z_{G}$. Let $Z^{\prime}=Z_{i+1}$ be the equivariant adjunction space

$$
Z^{\prime}=Z \cup_{\phi}\left(e^{2 m+1} \times G\right)
$$

and let $Z_{1}^{\prime} \subset Z^{\prime}$ be the ordinary adjunction space

$$
Z_{1}^{\prime}=Z \cup_{\chi} e^{2 m+1} .
$$

Then by Lemma 5 we can extend the maps $f, g$ to $Z_{1}^{\prime}$ and so we can extend them equivariantly to maps $f^{\prime}: Z^{\prime} \rightarrow X$ and $g^{\prime}: Z^{\prime} \rightarrow Y$. Again we can extend the map $J$ to a $B_{G^{-m a p}} J^{\prime}: Z_{G}^{\prime} \rightarrow \tilde{Z}$. Therefore we get a 
commutative diagram

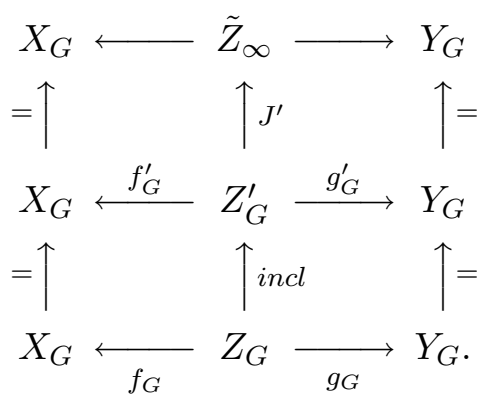

Moreover one has $\operatorname{dim}_{\mathbb{Q}} H^{*}\left(\tilde{Z}_{\infty}, Z_{G}^{\prime}\right)=\operatorname{dim}_{\mathbb{Q}} H^{*}\left(\tilde{Z}_{\infty}, Z_{G}\right)-1$, since the onedimensional subspace generated by $g^{\prime} \in H_{2 m+1}\left(M, Z_{G}\right)$ has been killed attaching the homotopy-cell $E_{G} \times{ }_{G}\left(e^{2 m+1} \times G\right)$ to $Z_{G}$. Substituting $Z:=$ $Z^{\prime}, J:=J^{\prime}, f:=f^{\prime}, g:=g^{\prime}$ we can iterate the process until $H^{*}\left(\tilde{Z}_{\infty}, Z_{G}\right)$ is zero, $J$ is a rational homotopy equivalence and $f$ and $g$ are equivariant maps inducing isomorphisms in rational cohomology.

\section{Special points in the spectrum of $H^{*} B a u t_{0}(X)$.}

Let $k$ be a field. Let $A_{0}=P / I_{0}$, where $P=k\left[X_{1}, \ldots, X_{n}\right]$ the graded commutative polynomial algebra in the indeterminates $X_{1}, \ldots, X_{n}$ of positive degree with $\operatorname{deg} X_{i} \equiv 0(\bmod 2), i=1, \ldots, n$. If $R$ is a positively graded $k$-algebra let $P_{R}=R\left[X_{1}, \ldots, X_{n}\right]$. The augmentation ideal of the positively graded ring $R$ will be denoted by $\underline{m}_{R}$.

Lemma 6. Let $R$ be a connected non-negatively graded $k$-algebra, let $I_{0} \subset$ $\underline{m}_{P} P$ be the ideal generated by homogeneous elements $f_{1}, \ldots, f_{m}$. Let $A_{0}=$ $P / I_{0}$ and assume $\operatorname{dim}_{k} A_{0}<\infty$. If $A$ is a graded $R$-algebra, free as a $R$ module such that $A / \underline{m}_{R} A$ is isomorphic to $A_{0}$ as a graded $k$-algebra, then $A$ can be written as $A=P_{R} / I$ where the ideal $I \subset P_{R}$ is generated by homogeneous elements $F_{j}$ of the form $F_{j}=f_{j}+r_{j}, j=1, \ldots, m, r_{j} \in \underline{m}_{R} P_{R}$. Furthermore, $A$ is a finitely generated $R$-module with $r k_{R} A=\operatorname{dim}_{k} \overline{A_{0}}$.

For a proof see [7]. The $R$-algebra $A$ will be called a (positively) graded deformation of $A_{0}$ along the ring $R$. Here and in the following we put $k=\mathbb{Q}$. By the observations at the beginning of Section 2 the ring $H_{G}^{*}(X)$ is a deformation of $H^{*}(X)$ along $R_{G}$. It is clear that the polynomials $r_{j}$ are coding a certain amount of information on the geometric behaviour of the $G$-action. This information can be detected by the Borel-Quillen-Hsiang localization theorem. We want to characterize those defomations which arise as equivariant cohomology rings for circle actions on spaces $X$ where the $\mathbb{Q}$ algebra $A_{0}=H^{*}(X)$ is multiplicatively generated by finitely many elements $x_{1}, \ldots, x_{n}$ of degree $d_{i}=\operatorname{deg} x_{i}=2$. Let $R=\mathbb{Q}[t], \operatorname{deg} t=2$, and let $A=$ $P_{R} / I$, be a deformation of $A_{0}$ along $R$. If $A_{0}=P / I_{0}$ where $I_{0} \subset\left(x_{1}, \ldots, x_{n}\right)$ 
is generated by the homogeneous polynomials $f_{1}, \ldots, f_{n}$, then by Lemma 6 the ideal $I$ has a generator system of the type $F_{j}, j=1, \ldots, n, F_{j}=$ $f_{j}+r_{j}, r_{j} \in(t) P_{R}$. The following definition is motivated by Thm. 2 and Lemma 2 which give necessary conditions for the structure of the exact sequence of the pair $\left(X_{G}, X_{G}^{G}\right)$.

Definition 4. A graded deformation $A$ of $A_{0}$ along $R$ will be called a gdeformation if the following is true: Let $(0)=\cap_{\alpha} Q_{\alpha}$ be the primary decomposition of the zero ideal in $A$ then the corresponding exact chinese sequence

$$
0 \rightarrow A \rightarrow \prod_{\alpha} A / Q_{\alpha} \rightarrow C \rightarrow 0
$$

is a $\mathbf{g}$-sequence. (Def. 2.)

In particular there are graded artinian $k$-algebras $B_{\alpha}$ such that $A / Q_{\alpha} \cong$ $R \otimes_{k} B_{\alpha}$. By graduation reasons, the algebras $B_{\alpha}$ are also multiplicatively generated over $\mathbb{Q}$ by elements of degree two. According to Thm. 2 (iii) the associated prime ideals $P_{\alpha}=\sqrt{Q_{\alpha}}$ of $Q_{\alpha}$ are regular and therefore have the form

$$
P_{\alpha}=\left(X_{1}-\omega_{\alpha, 1}, \ldots, X_{n}-\omega_{\alpha, n}\right),
$$

where $\omega_{\alpha, i}=w_{\alpha, i} t, w_{\alpha, i} \in \mathbb{Q}$. For a geometric interpretation of the numbers $w_{\alpha, i}$ in the case $A$ is the equivariant cohomology of a smooth circle action on a smooth manifold, see e.g. [7], Th. 2.2.

Let $X$ be a topological space, then denote by $\operatorname{aut}_{0}(X)$ the topological monoid of those homotopy self equivalences $h: X \rightarrow X$ which are homotopic to the identity map. Choose a base point $\{*\} \subset X$ and consider the corresponding submonoid $\operatorname{aut}_{0}^{\bullet}(X) \subset \operatorname{aut}_{0}(X)$ of pointed self homotopy equivalences. The fibration

$$
\operatorname{aut}_{0}^{\bullet}(X) \rightarrow \operatorname{aut}_{0}(X) \rightarrow X
$$

gives rise to a fibration $\xi_{u}$

$$
X \rightarrow \operatorname{Baut}_{0}^{\bullet}(X) \rightarrow \text { Baut }_{0}(X) .
$$

which is universal for the functor of oriented fiber homotopy classes of oriented Hurewicz fibrations with fiber $X$, see e.g., [8], Thm. 3.3. Applying the cohomology functor gives a commutative diagram

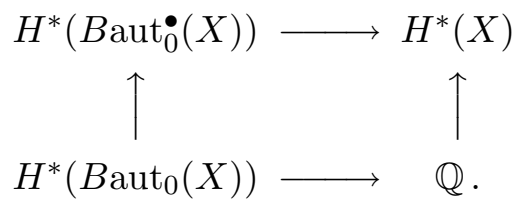

If $X$ is a space of type $\mathrm{F}$ such that $H^{*}(X)$ has no odd cohomology and $A_{0}=H^{*}(X)$ has no negative derivations, the diagram (28) represents the positively graded part of a graded versal deformation of the $\mathbb{Q}$-algebra $A_{0}=$ 
$H^{*}(X)$, see [8], no. 3 . In the following this part will be called $\theta_{u}$. Moreover in [8] it is shown that $\theta_{u}$ has even the universality (!) property in so much as any positively graded deformation $\gamma=\left\{S \rightarrow A \rightarrow A_{0}\right\}$ over a positively graded ring $S$ is given by a base change with a unique homomorphism $f_{\gamma}$ : $H^{*}\left(\operatorname{Baut}_{0}(X)\right) \rightarrow S$.

Let $S=R=\mathbb{Q}[t]$, deg $t=2$, and let $L=\operatorname{Spec} R=\operatorname{Hom}(R, k)$ be the weighted line. Let $V=\operatorname{Spec} H^{*}\left(B \operatorname{But}_{0}(X)\right)$ then to $f_{\gamma}$ corresponds a geometric morphism $\nu: L \rightarrow V$. Let $G_{m} \cong \mathbb{Q}^{*}$. The image of $L$ under $\nu$ is a $G_{m}$-stable curve $C, 0 \in C$, which in general will have a singularity in the origin. Thus we can consider $C$ as the closure of the $G_{m}$-orbit of one of its $\mathbb{Q}$-valued points $t \in C-\{0\}$, i.e., $C=\operatorname{cl}\left(G_{m} \cdot t\right)$ which as a set is equal to $G_{m} \cdot t \cup\{0\}$. Then $C$ is a monomial curve with a $G_{m}$-equivariant normalization $\nu: L \rightarrow C$ given by a ring inclusion of the type

$$
\mathbb{Q}\left[a_{1} t^{m_{1}}, \ldots, a_{s} t^{m_{s}}\right] \subset \mathbb{Q}[t] .
$$

Conversely, let $t \in V$ be a $\mathbb{Q}$-valued (and thus closed) point, let $C_{t} \subset V$ be the closure of its $G_{m}$-orbit and let $\nu_{t}: L \rightarrow C_{t}$ be the corresponding $G_{m}$-equivariant normalization. Let $\gamma_{t}$ be the induced $G_{m}$-equivariant deformation on the weighted line, i.e., $\gamma_{t}=\nu_{t}^{*} \theta_{u}$.

Definition 5. A $\mathbb{Q}$-valued point $t$ in the spectrum of $H^{*}\left(\operatorname{Baut}_{0}(X)\right)$ is called a g-point, if $\gamma_{t}$ is a $\mathbf{g}$-deformation.

Obviously any equivariant cohomology ring of a circle action on $X$ gives rise to a g-point in $V$. Conversely to any g-point in $V$ there exists a circle action on a space $Y$ with $H^{*}(Y) \cong A_{0}$ as follows from the realization theorem in $[\mathbf{1 3}]$.

Denote by $\Gamma\left(A_{0}\right)$ the group of graded automorphisms of the $\mathbb{Q}$-algebra $A_{0}$. Then $\Gamma\left(A_{0}\right)$ contains the grading group $\Gamma_{0} \cong G_{m}=\mathbb{Q}^{*}$ as a central subgroup. Moreover suppose $\Gamma\left(A_{0}\right)$ is reductive over $\mathbb{Q}$. (This for example is true for the cohomology algebra of the flag variety $X=K / T, K$ a compact Lie group, $T \subset K$, a maximal torus where the group $\Gamma\left(A_{0}\right) / \Gamma_{0}$ is given by the symmetry group of the root system of $K$ and therefore is also a finite group, see e. g. [11].) As it is well-known in deformation theory, the $\Gamma\left(A_{0}\right)$ action on $A_{0}$ induces an action on the deformation $\theta_{u}$, see e.g., [14]. Thus the space $V$ inherits a structure of a representation space of the group $\Gamma\left(A_{0}\right)$. Denote by $V\left(G ; A_{0}\right) \subset V$ the subset of $\mathbf{g}$-points of $V$. Then the $\Gamma\left(A_{0}\right)$-action on $V$ induces a $\Gamma\left(A_{0}\right)$-action on $V\left(G ; A_{0}\right)$. Denote by

$$
P\left(G ; A_{0}\right)=\left(V\left(G ; A_{0}\right)-\{0\}\right) / \Gamma\left(A_{0}\right)
$$

the corresponding quotient space.

Recall that an action of a compact Lie group $G$ on $X$ is called c-effective with coefficients in $k$ if $H^{*}\left(X, X^{S} ; k\right) \neq 0$ for every subtorus $S \subset G$. In the following we say that an action is c-effective if it is c-effective with coefficients in $\mathbb{Q}$. In the case we consider here, i.e., if $H^{*}(X)$ is generated by elements 
of degree two, and if $G$ is the circle, an action is c-effective if and only if $H_{G}^{*}(X)$ is not isomorphic to $H^{*}(X) \otimes_{\mathbb{Q}} R_{G}$ as a graded $R_{G}$-algebra as follows easily from Sec. 3.

Define now

$$
O p^{\mathbb{Q}}\left(G ; A_{0}\right)
$$

as the set of equivariant connected $\mathbb{Q}$-homotopy types of c-effective $G$-actions with L-property on $F$-spaces $Y$ with $H^{*}(Y) \cong A_{0}$ with componentwise simply connected fixed spaces.

Let $\bar{\phi} \in O p^{\mathbb{Q}}\left(G ; A_{0}\right)$ and take a representative $\phi: G \times Y \rightarrow Y$ of $\bar{\phi}$. The Borel construction gives a fibration

$$
\beta_{\phi}: X \rightarrow E_{G} \times_{G} Y \rightarrow B_{G}
$$

which in turn determines up to homotopy a classifying map $f_{\phi}: B_{G} \rightarrow$ $\operatorname{Baut}_{0}(X)$ and thus a homomorphism $H^{*}\left(f_{\phi}\right): H^{*}\left(\operatorname{Baut}_{0}(X)\right) \rightarrow H^{*}\left(B_{G}\right)$, which gives a point in $P\left(G ; A_{0}\right)$. We thus get a map

$$
\omega: O p^{\mathbb{Q}}\left(G ; H^{*}(X)\right) \rightarrow P\left(G ; H^{*}(X)\right) .
$$

Theorem 5. Let $A_{0}=H^{*}(X ; \mathbb{Q})$ be a complete intersection of finite length generated by elements of degree 2 with $\Gamma\left(A_{0}\right)$ reductive. Then the map $\omega$ is bijective.

Before we come to the proof of Theorem 5 we have to collect some preliminary results.

Lemma 7. Let $A_{0}$ be a graded complete intersection over $\mathbb{Q}$ of finite length generated by elements of degree two. Let $R=\mathbb{Q}[t], \operatorname{deg} t=2$ and let $A$ and $A^{\prime}$ be $\mathbf{g}$-deformations of $A_{0}$ along $R$. Let $\phi: A \rightarrow A^{\prime}$ be a graded $R$ isomorphism. If $(0)=\cap_{i \in I} Q_{i}$ and $(0)^{\prime}=\cap_{j \in J} Q_{j}^{\prime}$ are the respective primary decompositions of the zero ideals in $A, A^{\prime}$ respectively, then $I=J$ and there is a permutation $\pi$ and homomorphisms $\phi_{i}$ such that there is a commutative diagram

$$
\begin{aligned}
& 0 \rightarrow A \quad \longrightarrow \quad \prod_{i} A / Q_{i} \\
& \phi \downarrow \quad \prod_{i} \phi_{i} \\
& 0 \rightarrow A^{\prime} \quad \longrightarrow \prod_{i} A^{\prime} / Q_{\pi(i)}^{\prime}
\end{aligned}
$$

where the $\phi_{i}$ are the induced isomorphisms.

Proof. We have $(0)^{\prime}=\phi(0)=\phi\left(\cap_{i} Q_{i}\right)=\cap_{i} \phi\left(Q_{i}\right)=\cap_{j} Q_{j}^{\prime}$. Since the rings $A$ and $A^{\prime}$ are Cohen-Macaulay the zero ideals are unmixed. Therefore they have a unique (up to permutation) primary decomposition. This shows $I=J$ and $\phi\left(Q_{i}\right)=Q_{\pi(i)}^{\prime}$ for a permutation $\pi$. Let $\phi_{i}: A / Q_{i} \rightarrow A / Q_{\pi(i)}^{\prime}$ be the induced homomorphisms, then the above diagram is commutative. 
Lemma 8. Let $\left.A_{0}\right|_{\mathbb{Q}}$ be a complete intersection with $\Gamma\left(A_{0}\right)$ reductive and let $s, t \in V$ be $\mathbb{Q}$-valued points in the base $V$ of $\theta_{u}$ with $s=\gamma t, \gamma \in \Gamma\left(A_{0}\right)$. Then the corresponding graded 1-parameter deformations $\nu_{t}^{*} \theta_{u}$ and $\nu_{s}^{*} \theta_{u}$ are isomorphic as $\mathbb{Q}[t]$-algebras.

This is obvious.

Proof of Theorem 5. The surjectivity of the map $\omega$ is guaranteed by the main result of $[\mathbf{1 3}]$.

Suppose $\left(X_{1}, \phi\right)$ and $\left(X_{2}, \psi\right)$ are $S^{1}$-actions of the kind considered here on spaces $X_{1}$ and $X_{2}$ with the rational cohomology of $X$. Let $s=\omega(\phi)$ and $t=\omega(\psi)$ be the corresponding g-points. Let $\gamma \in \Gamma\left(A_{0}\right)$ with $s=\gamma t$. Then by Lemma 7 the corresponding 1-parameter deformations $\nu_{t}^{*} \theta_{u}$ and $\nu_{s}^{*} \theta_{u}$ are isomorphic as $\mathbb{Q}[t]$-algebras. This means that the equivariant cohomology rings $H_{\psi}^{*}(X)$ and $H_{\phi}^{*}(X)$ are isomorphic as $R_{G^{-}}$algebras. By Lemma 6 the actions are similar. By Theorem 4 the actions are equivariantly $\mathbb{Q}$-homotopy equivalent which proves injectivity.

\section{Applications.}

In the following we want to give a more intrinsic criterion for a point $t \in V$ to be a $\mathrm{g}$-point.

Definition 6. Let $k$ be a field, let $P=k\left[x_{1}, \ldots, x_{n}\right]$ and let $f \in P$. Let $w=\left(w_{1}, \ldots, w_{n}\right) \in k^{n}$ and put $f^{w}\left(x_{1}, \ldots, x_{n}\right)=f\left(x_{1}+w_{1}, \ldots, x_{n}+w_{n}\right)$.

(i) The polynomial $f$ is called translation homogeneous if there exists a vector $w \in k^{n}$ such that $f^{w}$ is homogeneous.

(ii) An ideal $J \subset P$ is called translation homogeneous if there exists a generator system $f_{1}, \ldots, f_{m}$ of $J$ and a $w \in k^{n}$ such that $f_{1}^{w}, \ldots, f_{m}^{w}$ are homogeneous.

Proposition 1. Let $J \subset P$ be an ideal generated by a regular series of maximal length, let $J^{h} \subset k\left[x_{0}, \ldots, x_{n}\right]$ be its homogeneization with respect to $x_{0}$. Then the following statements are equivalent.

(i) There is a graded complete intersection $B_{0}$ and a graded isomorphism

$$
k\left[x_{0}, \ldots, x_{n}\right] / J^{h} \cong k\left[x_{0}\right] \otimes_{k} B_{0}
$$

of $k\left[x_{0}\right]$-algebras.

(ii) The ideal $J \subset k\left[x_{1}, \ldots, x_{n}\right]$ is translation homogeneous.

For the proof of this proposition we need the following statement on homogeneous ideals in the polynomial ring $P$.

Proposition 2. Let $k$ be a field, char $k=0$, let $I \subset P$ be an ideal such that the following conditions are satisfied. 
(i) The ideal $J$ is generated over $P$ by a regular series of maximal length $n$.

(ii) The ideal $J$ is generated by homogeneous elements.

Then $J$ is generated by a regular series of homogeneous elements of maximal length $n$.

Proof. Let $A=P / J$ and consider the $A$-module $J / J^{2}$. Then by (i) $J / J^{2}$ is free of rank $n$. By (ii) it is graded, i.e., has a $k^{*}$-action induced by the $k^{*}$ action on $P$ and $J$. The $n$-dimensional $k$-vector space $V=J / J^{2} \otimes_{A} k$ inherits a graduation in a natural way. Consider the natural projection $p: J \rightarrow V$. Since $k^{*}$ is reductive, there exists a series $f_{1}, \ldots, f_{n}$ of homogeneous elements in $J$ projecting to a graded base of $V$. Let $K$ be the subideal of $J$ generated by $f_{1}, \ldots, f_{n}$. Let $B=P / K$. By the choice of the $f_{j}$ the canonical map $c: K / K^{2} \otimes_{B} A \rightarrow J / J^{2}$ induces an isomorphism after applying $(-) \otimes_{A} k$. It follows that $c$ is surjective. Let $0 \rightarrow K \rightarrow J \rightarrow C \rightarrow 0$ be the exact sequence of $P$-modules given by the inclusion $K \subset J$. Then one has $K \otimes_{P} A=$ $K / K^{2} \otimes_{B} A$ and $J \otimes_{B} A=J / J^{2}$ and the homomorphism induced by the inclusion between these $A$-modules is equal to $c$. Thus it follows $C \otimes_{P} A=0$. In particular one has $C \otimes_{P} k=0$ and by the graded version of the Lemma of Nakayama it follows $C=0$. But then $K=J$. It is clear that the $f_{j}$ are a series of consecutive nonzero divisors since $\operatorname{dim} P / J=0$.

Proof of Proposition 1. (i) $\Rightarrow$ (ii): Let $B_{0}=P /\left(g_{1}, \ldots, g_{n}\right)$, where the $g_{j}$ are homogogeneous. By hypothesis we have a graded isomorphism of $k\left[x_{0}\right]$ algebras

$$
\alpha: k\left[x_{0}\right] \otimes_{k} \frac{P}{\left(g_{1}, \ldots, g_{n}\right)} \rightarrow \frac{k\left[x_{0}, \ldots, x_{n}\right]}{J^{h}}
$$

which by graduation reasons must be of the form

$$
\alpha\left(x_{i}\right)=\sum_{j} \alpha_{i j} x_{j}+\beta_{i} x_{0}, i=1, \ldots, n
$$

where $\left(\alpha_{i j}\right)$ is a non-singular $n \times n$-matrix with $k$-entries and $\beta_{i} \in k$. Let $v=\left(v_{1}, \ldots, v_{n}\right)$ be the solution of the system $\sum_{j} \alpha_{i j} v_{j}=\beta_{i}, i=1, \ldots, n$. Let $y_{i}=\sum_{j} \alpha_{i j}\left(x_{j}+v_{j} x_{0}\right)$, let $K \subset k\left[x_{0}, \ldots, x_{n}\right]$ be the ideal generated by the polynomials $g_{j}\left(y_{1}, \ldots, y_{n}\right), j=1, \ldots n$ Then $K \subset J^{h}$. We show that $K=J^{h}$.

By the inclusion of ideals we have the natural projection

$$
p: k\left[x_{0}, \ldots, x_{n}\right] / K \rightarrow k\left[x_{0}, \ldots, x_{n}\right] / J^{h}
$$

which remains surjectice after tensorizing over $k\left[x_{0}\right]$ with $k$. But the tensorized rings are of the same $k$-length and therefore $\bar{p}$ is an isomorphism. Now the ring $k\left[x_{0}, \ldots, x_{n}\right] / J^{h}$ is a free $k\left[x_{0}\right]$-module and therefore Ker $p \otimes_{k\left[x_{0}\right]}$ 
$k=\operatorname{Ker} \bar{p}$. This shows $\operatorname{Ker} p \otimes_{k\left[x_{0}\right]} k=0$ and therefore by the graded version of the Lemma of Nakayama it follows Ker $p=0$. So, $p$ must be an isomorphism and $K=J^{h}$.

But then $J^{h}=\left(F_{1}, \ldots, F_{n}\right)$ where the $F_{j}=g_{j}\left(y_{1}, \ldots, y_{n}\right)$ are the homogeneizations of the polynomials

$$
f_{i}^{v}=g_{i}\left(\sum_{j} \alpha_{i j}\left(x_{j}+v_{j}\right), \ldots, \sum_{j} \alpha_{n j}\left(x_{j}+v_{j}\right)\right)
$$

with respect to $x_{0}$, i.e., $J=\left(f_{1}^{v}, \ldots, f_{n}^{v}\right)$ with

$$
f_{i}=g_{i}\left(\sum_{j} \alpha_{i j} x_{j}, \ldots, \sum_{j} \alpha_{n j} x_{j}\right) .
$$

But of course the $f_{i}$ are homogeneous with respect to $x_{1}, \ldots, x_{n}$, i.e., $J$ is translation homogeneous.

(ii) $\Rightarrow$ (i): Let $J=\left(f_{1}^{v}, \ldots, f_{m}^{v}\right)$ where $f_{1}, \ldots, f_{m}$ is a series of homogeneous elements. Put $J_{0}=\left(f_{1}, \ldots, f_{m}\right)$. Since $J$ is generated by a regular series of elements of maximal length, the same is true for $J_{0}$. Thus it follows by Proposition 2 that $J_{0}$ is generated by a regular series of maximal length of homogeneous elements which by simplicity will be called $g_{1}, \ldots, g_{n}$. It follows $J=\left(g_{1}^{v}, \ldots, g_{n}^{v}\right)$. Let $F_{j}$ be the homogeneization of $g_{j}^{v}$ with respect to $x_{0}$. Then the homogeneization $J^{h}$ of $J$ is given by $J^{h}=\left(F_{1}, \ldots, F_{n}\right)$, i.e., is given elementwise. This follows from the flatness of the $k\left[x_{0}\right]$-algebra $k\left[x_{0}, \ldots, x_{n}\right] /\left(F_{1}, \ldots, F_{n}\right)$ since the $g_{j}$ are a regular series. But then it follows easily that the map defined by $x_{i} \mapsto x_{i}-v_{i} x_{0}$ is an isomorphism $k\left[x_{0}, \ldots, x_{n}\right] / J^{h} \rightarrow k\left[x_{0}\right] \otimes_{k} B_{0}$, where $B_{0}=P /\left(g_{1}, \ldots, g_{n}\right)$.

In the following we write $\theta_{u}=\left\{A(V) \rightarrow A(X) \rightarrow A_{0}\right\}$. The geometric notation is $\theta_{u}=\left\{X_{0} \rightarrow X \rightarrow V\right\}$. Let $t \in V$ be a $\mathbb{Q}$-valued point and let $X_{t}$ be the fiber of $t$. Let $X_{t}=X_{t, 1} \sqcup \cdots \sqcup X_{t, \rho}$ be the decomposition of $X_{t}$ into irreducible (but not necessarily reduced) components. If $A(X)=A(V)\left[x_{1}, \ldots, x_{n}\right] / I$, then the ring $A\left(X_{t}\right)$ has the form $A\left(X_{t}\right)=$ $\mathbb{Q}(t)\left[x_{1}, \ldots, x_{n}\right] / I_{t}$, where $\mathbb{Q}(t) \cong \mathbb{Q}$ is the residue field of $t$ and $I_{t}$ the defining ideal of $X_{t}$. Let $I_{t}=\cap_{j} I_{t, j}$ be the corresponding primary decomposition.

Lemma 9. $A \mathbb{Q}$-valued point $t \in V$ is a $\mathbf{g}$-point if and only if for every $j$ the ideal $I_{t, j}$ is translation homogeneous.

Thus it follows that for every $j$ there exists a $w_{j} \in \mathbb{Q}^{n}$ such that $\sqrt{I_{t, j}}=$ $m_{j}=\left(x_{1}-w_{j 1}, \ldots, x_{n}-w_{j n}\right)$.

Proof. The condition is necessary. This follows from the above proposition applied to the primary components $I_{t, j}$. 
The condition is sufficient. It is easy to see that the induced deformation $\gamma_{t}$ is given by the homogeneization of $A\left(X_{t}\right)$, i.e., let the variable $x_{0}$ be denoted by $x_{0}=s$, then denote by $I_{t}^{h} \subset \mathbb{Q}\left[s, x_{1}, \ldots, x_{n}\right]$ the homogeneization of $I_{t}$ with respect to $s$. Moreover let

$$
A=\mathbb{Q}\left[s, x_{1}, \ldots, x_{n}\right] / I_{t}^{h} .
$$

The primary decomposition of $I_{t}$ gives a primary decomposition

$$
I_{t}^{h}=\bigcap_{j} I_{t, j}^{h}
$$

By the above proposition the algebras

$$
A_{j}=\mathbb{Q}\left[s, x_{1}, \ldots, x_{n}\right] / I_{t, j}^{h}
$$

are in the graded sense isomorphic to $\mathbb{Q}[s] \otimes_{\mathbb{Q}} B_{j}$, where the $B_{j}, j=1, \ldots, \rho$, are graded $\mathbb{Q}$-algebras of finite length, generated by $x_{1}, \ldots, x_{n}$. Therefore the exact sequence

$$
0 \rightarrow A \rightarrow \prod_{j} A_{j}
$$

is equivalent to

$$
0 \rightarrow A \rightarrow \prod_{j} R \otimes_{\mathbb{Q}} B_{j}
$$

with $R=\mathbb{Q}[s]$.

As a first example we consider the versal $G_{m}$-equivariant deformation of the graded $\mathbb{Q}$-algebra $A_{0}=\mathbb{Q}[x] /\left(x^{n+1}\right)$. The usual exact sequence [8], no. 1 , gives for $f=x^{n+1}$ the expression

$$
T^{1}\left(A_{0}\right) \cong \frac{\partial}{\partial f} A_{0} / x^{n} A_{0}
$$

Thus it follows that $T^{1}\left(A_{0}\right)$ is a $\mathbb{Q}$-vector space of dimension $n$. Therefore the versal deformation has the form $\theta_{u}=\left\{R \rightarrow A \rightarrow A_{0}\right\}$ with $R=\mathbb{Q}\left[t_{2}, \ldots, t_{n+1}\right]$ and $A=R[X] /(F)$ where $F$ is given by

$$
F(X)=X^{n+1}+t_{2} X^{n-1}+\cdots+t_{n+1} .
$$

Let $V=\mathbb{Q}^{n+1}$ be the set of $\mathbb{Q}$-valued points of Spec $R$, let $\sigma_{1}, \sigma_{2}, \ldots, \sigma_{n+1}$ be the elementary symmetric polynomials.

Lemma 10. The $\mathbf{g}$-points are precisely the points $s=\left(s_{1}, \ldots, s_{n+1}\right) \in V$ with

$$
\begin{array}{r}
s_{i}=\sigma_{i}\left(w_{1}, \ldots, w_{n+1}\right) \\
\text { for } w=\left(w_{1}, \ldots, w_{n+1}\right) \in \mathbb{Q}^{n+1} \text { and } \sum_{i} w_{i}=0 .
\end{array}
$$


Proof. It is clear that the points of the described type are g-points. Conversely, let $s \in V$, be a g-point. The corresponding fiber algebra is given by $A_{s}=\mathbb{Q}(s)[X] /\left(F_{s}\right)$ with $F_{s}=x^{n+1}+s_{2} x^{n-1}+\cdots+s_{n+1}$. By the definition of a $\mathrm{g}$-point $F_{s}$ must split into a product of linear factors, i.e.,

$$
F_{s}(X)=\prod_{j=1}^{\rho}\left(X-w_{j}\right)^{m_{j}},
$$

$\sum_{j} m_{j}=n+1$. Moreover the polynomial $F_{s}$ does not have a $n$-power if and only if $\sum_{j} w_{j}=0$. This shows that the coefficients have the desired form. The converse is clear.

Recall that the cohomology $H^{*}(X)$ of the complex projective space $X=$ $\mathbb{C P}^{n}$ is given by $A_{0}$. If $K$ is a circle acting on $X$ such that the L-property is satisfied, then the corresponding equivariant cohomology is given by the homogeneization of a ring of type $A_{s}$, i.e.,

$$
H_{K}^{*}(X) \cong H^{*}\left(B_{K}\right)[\xi] /(F)
$$

with

$$
F=\prod_{j=1}^{\rho}\left(\xi-w_{j} t\right)^{m_{j}}
$$

If we put

$$
F_{j}=\left(\xi-w_{j} t\right)^{m_{j}},
$$

the exact sequence in Th. 2 (i) assumes the form

$$
0 \longrightarrow H^{*}\left(B_{K}\right)[\xi] /(F) \longrightarrow \prod_{j=1}^{\rho} H^{*}\left(B_{K}\right)[\xi] /\left(F_{j}\right) .
$$

This is the exact sequence for equivariant cohomology considered in Sec. 3 . In the case of a circle action on a space $X$ with the rational cohomology of $\mathbb{C P}^{n}$. In the following this exact sequence will be denoted with $C h\left(w_{1}, \ldots, w_{n+1}\right)$. The $(n+1)$-tuple $\left(w_{1}, \ldots, w_{n+1}\right)$ is called the (rational) weight system of the action.

Remarks 2. If a circle acts linearly on $\mathbb{C P}^{n}$ induced by a representation with diagonal matrix

$$
\rho(\exp (2 \pi \sqrt{-1} t))=\operatorname{diag}\left\{\exp \left(2 \pi \sqrt{-1} w_{1} t\right), \ldots, \exp \left(2 \pi \sqrt{-1} w_{\rho} t\right)\right\},
$$

where the weights $w_{j} \in \mathbb{Z}$ have multiplicity $m_{j}$, then the roots of $F$ can be identified with the $w_{j} t$.

We make now a direct application of Th. 4 establishing the automorphisms of such an exact sequence. The $R_{K}$-automorphisms of $H_{K}^{*}(X)$ are of the form $\xi \mapsto \mu \xi+\lambda t$ with $\lambda \in \mathbb{Q}$ and $\mu \in \mathbb{Q}^{*}$. These maps induce automorphisms of the chinese sequence (30) in the sense of Definition 3. Conversely, 
suppose there is given a similarity between two exact sequences of the type (30):

$$
\begin{aligned}
& H^{*}\left(B_{K}\right)[\xi] /(F) \longrightarrow \prod_{j=1}^{\rho} H^{*}\left(B_{K}\right)\left[\eta_{j}\right] /\left(\left(\eta_{j}-v_{j} t\right)^{m_{j}}\right) \\
& \alpha \downarrow \downarrow \beta \\
& H^{*}\left(B_{K}\right)[\xi] /(G) \longrightarrow \prod_{j=1}^{\rho} H^{*}\left(B_{K}\right)\left[\eta_{j}\right] /\left(\left(\eta_{j}-w_{j} t\right)^{n_{j}}\right) .
\end{aligned}
$$

Then $\alpha$ has been induced by a homomorphism of the type $\xi \mapsto \mu \xi+\lambda t$. At the other hand the isomorphism $\beta$ includes only permutation of factors and eventually for every factor the multiplication with a $\nu_{j} \in \mathbb{Q}^{*}$. The permutations don't affect the automorphism $\alpha$. Moreover by commutativity the $\nu_{j}$ must all be equal. Therefore the group $\mathcal{S}$ of similarities of a sequence $C h\left(w_{1}, \ldots, w_{n+1}\right)$ can be described as follows. Let $V \cong \mathbb{Q}^{n+1}$ then consider the group $\mathcal{S}=T \rtimes E \times \mathcal{S}_{n+1}$ where $T \cong \mathbb{Q}$ is the group of diagonal translations and $E \cong \mathbb{Q}^{*}$ is the group of homotheties whereas the symmetric group $\mathcal{S}_{n+1}$ is acting on $V$ by permutation of coordinates. Now $\mathcal{S}$ is acting in the following way on the set of exact chinese sequences of the type (30): Let $\Sigma=(\lambda, \mu, \pi) \in \mathcal{S}$ then

$$
C h\left(w_{1}, \ldots, w_{n}\right)^{\Sigma}=C h\left(\mu\left(w_{\pi(1)}+\lambda\right), \ldots, \mu\left(w_{\pi(n+1)}+\lambda\right)\right) .
$$

Therefore we conclude:

Lemma 11. The exact sequences $C h\left(v_{1}, \ldots, v_{n+1}\right)$ and $C h\left(w_{1} \ldots, w_{n+1}\right)$ are similar if and only if there exists a $\Sigma=(\lambda, \mu, \pi) \in \mathcal{S}$ with

$$
\left(v_{1}, \ldots, v_{n+1}\right)=\left(\mu\left(w_{\pi(1)}+\lambda\right), \ldots, \mu\left(w_{\pi(n+1)}+\lambda\right)\right) .
$$

Then by Th. 4 we get the following nonsurprising result.

\section{Theorem 6.}

(i) Any circle action on a 1 -connected space $X$ with $H^{*}(X) \cong \mathbb{Q}[x] /\left(x^{n+1}\right)$ which satisfies the L-property and has a componentwise simply connected fixed space is $\mathbb{Q}$-equivariantly homotopy equivalent to a linear action on a $\mathbb{C P}^{n}$.

(ii) Two such circle actions with weight systems $\left\{v_{i}\right\}$ and $\left\{w_{i}\right\}$ are $\mathbb{Q}$ equivariantly homotopy equivalent if and only if there exists a $\lambda \in \mathbb{Q}$, $a \mu \in \mathbb{Q}^{*}$ and a permutation $\pi$ such that

$$
\left(v_{1}, \ldots, v_{n+1}\right)=\left(\mu\left(w_{\pi(1)}+\lambda\right), \ldots, \mu\left(w_{\pi(n+1)}+\lambda\right)\right) .
$$

The quotient $V / T$ with respect to the translation group can be identified with the subspace $U=\left\{\left(w_{1}, \ldots, w_{n+1}\right) \mid \sum_{i} w_{i}=0\right\}$ on which the restgroup $E \times \mathcal{S}_{n+1}$ acts in a natural way. Therefore the set of similarity classes of exact chinese sequences can be identified with the fundamental domain of the action of the Weyl group $W(S U(n+1))$ on the set of rational points of the Cartan subalgebra of $s u(n+1)$. This will be called the rationalized 
fundamental Weyl chamber or $F W_{n}(\mathbb{Q})$. Now it remains to divide out the natural action of $E \cong \mathbb{Q}^{*}$. Thus we have by Th. 4 .

\section{Theorem 7.}

$$
O p^{\mathbb{Q}}\left(S^{1}, \mathbb{C P}^{n}\right) \cong F W_{n}(\mathbb{Q}) / \mathbb{Q}^{*} .
$$

Thus we see that up to equivariant rational homotopy equivalence there is only one circle action on $S^{2}$, i.e., $O p^{\mathbb{Q}}\left(S^{1}, \mathbb{C P}^{1}\right)$ consists of a single point. The set $O p^{\mathbb{Q}}\left(S^{1}, \mathbb{C P}^{2}\right)$ can be identified with the set of rational numbers of a semiclosed intervall and $O p^{\mathbb{Q}}\left(S^{1}, \mathbb{C P}^{3}\right)$ is realized by the rational points of an equilateral triangle with two semiclosed edges omitted.

Remarks 3. (i) It is plausible that the homotopy type of the circle action on $\mathbb{C P}^{n}$ does only depend on the weight systems modulo a translation group isomorphic to $\mathbb{Z}$ since the weight system describes the linear action on the vector space $\mathbb{C}^{n+1}-\{0\}$ whereas the action on $\mathbb{C P}^{n}$ does only depend on the differences of weights.

(ii) The space $F W_{n}(\mathbb{Q}) / \mathbb{Q}^{*}$ can be obtained taking lattice vectors $\left(w_{1}, \ldots\right.$, $\left.w_{n+1}\right) \in \mathbb{Z}^{n+1}$ and then divide out the multiplicative group just giving a fundamental domain of the natural action of $W(S U(n+1))$ on the rational points of the projective space $P(\underline{h})$ of the Cartan subalgebra. But this is a quotient of the set of irreducible representations of $S U(n+$ 1).

(iii) In any case we see that the rational homotopy classification of arbitray circle actions on a space $X$ which is rationally homotopy equivalent to $\mathbb{C P}^{n}$ does not go beyond the corresponding classification of linear circle actions on $\mathbb{C P}^{n}$.

As another example we want to consider the case of the flag manifolds $X=K / T, K$ a compact connected Lie group and $T \subset K$ a fixed maximal torus. First we have to calculate the equivariant cohomology of a circle action on the homogeneous space $K / T$. Here we consider a slightly more general case. Let $K$ be a compact connected Lie group, $U \subset K$ be a closed connected subgroup and let $G$ be another compact Lie group, let $\rho: G \rightarrow K$ be a homomorphism. Then there is an action of of $G$ on $X=K / U$ by left translation:

$$
\begin{gathered}
\phi_{\rho}: G \times X \rightarrow X, \\
(g, k U) \rightarrow \rho(g) k U .
\end{gathered}
$$

Such actions will be called homomorphic or linear. We specialize to the case where $U \subset K$ is a closed connected subgroup of maximal rank. We put

$$
R_{G}=H^{*}\left(B_{G}\right), R_{U}=H^{*}\left(B_{U}\right), R_{K}=H^{*}\left(B_{K}\right) .
$$


Let $j: U \rightarrow K$ be the inclusion homomorphism and let $B j$ and $B \rho$ be the induced maps on the classifying spaces. Then there is a cartesian diagram

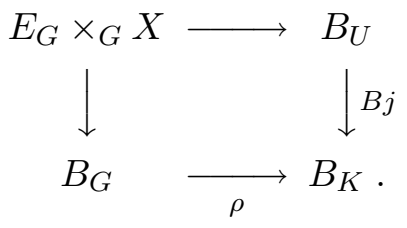

Since $U$ is a subgroup of maximal rank the ring homomorphism $(B j)^{*}$ : $R_{K} \rightarrow R_{U}$ makes the ring $R_{U}$ into a free $R_{K}$-module. Consequently the 2 -term of the corresponding Eilenberg-Moore spectral sequence is given by

$$
E_{2} \cong \operatorname{Tor}^{R_{K}}\left(R_{G}, R_{U}\right) \cong R_{G} \otimes_{R_{K}} R_{U},
$$

i.e., the higher Tor's vanish. Therefore the canonical homomorphism

$$
R_{G} \otimes_{R_{K}} R_{U} \rightarrow H_{G}^{*}(X)
$$

becomes an isomorphism of rings. Thus we can state the following suggestive result.

Theorem 8. Let $G$ be a compact, not necessarily connected Lie group and let $\rho: G \rightarrow K$ be a homomorphism into a compact connected Lie group $K$. Let $U \subset G$ be a closed connected subgroup of maximal rank. If $\phi_{\rho}: G \times X \rightarrow$ $X$ is the corresponding linear action on $X=K / U$ then there is a canonical isomorphism

$$
H_{G}^{*}(X) \cong R_{G} \otimes_{R_{K}} R_{U} .
$$

As an example take $G=U=T$ a fixed maximal torus of $K$. Then we obtain $H_{G}^{*}(X) \cong R_{G} \otimes_{R_{K}} R_{U} \cong R_{T} \otimes_{R_{K}} R_{T}$. In the following we want to investigate in a greater detail the structure of this ring and the corresponding geometric scheme.

Let $g$ be the real Lie algebra of $G$, denote by $\underline{h} \subset g$ the $\mathbb{R}$-Lie algebra of $T$. Let $\overline{\mathcal{O}}_{h}=\mathbb{Q}\left[\underline{h}^{*}\right]$ be the ring of rational valued regular functions on $\underline{h}$. Let $W=W_{G}=N_{G} / T$ be the Weyl group of $G$. Since $W$ acts $\mathbb{Q}$-rationally on $\underline{h}$ it acts naturally on the ring $\mathcal{O}_{\underline{h}}$. Denote by $\mathcal{O}_{h}^{W} \subset \mathcal{O}_{\underline{h}}$ the corresponding invariant ring. Now $W$ acts on $\underline{h}$ as a reflection group and then by the famous theorem of Shephard and Todd, see e.g., [4], Thm. 6.4.12, the invariant ring $\mathcal{O}_{h}^{W}$ is again a polynomial algebra. Since the natural homomorphism $R_{G} \rightarrow R_{T}$ induced by the fiber map $B_{T} \rightarrow B_{G}$ can be identified with the inclusion of homomorphism $\mathcal{O}_{\underline{h}}^{W} \subset \mathcal{O}_{\underline{h}}$, the pushout diagram

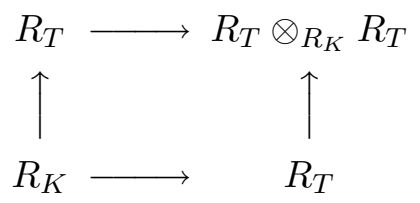


corresponds to the pullback diagram

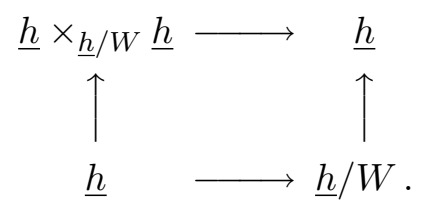

As a corollary of Th. 8 we obtain:

Lemma 12. The equivariant cohomology $H_{T}^{*}(K / T)$ of the natural $T$-action on $K / T$ is induced from the quotient morphism $\pi: h \rightarrow h / W$ by the same morphism.

There is then a unique homomorphism

$$
f_{\xi}: H^{*}\left(\operatorname{Baut}_{0}(K / T)\right) \rightarrow H^{*}\left(B_{K}\right)
$$

inducing the deformation $\xi$ :

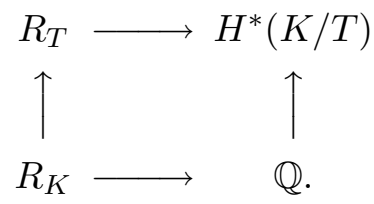

Let $R X=H^{*}\left(\operatorname{Baut}_{0}(X)\right)$ and $R^{\bullet} X=H^{*}\left(\operatorname{Baut}_{0}^{\bullet}(X)\right)$. We want to identify the deformation $\xi$ with a subdeformation of the universal deformation $\theta_{u}$.

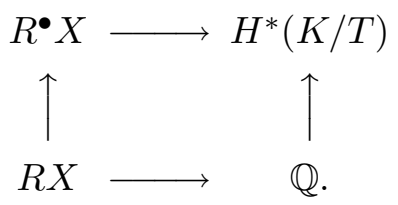

Let $V=\operatorname{Spec} R X$. Since $W=W K$ acts in a natural way on $R X$ it acts also on the space $V$. Let $Y=F i x(W, V)$ be the fixed space of this $W$-action. Let $S=\mathcal{O}_{Y}$ be the coordinate ring of $Y$.

Lemma 13. Let $K$ be a semisimple compact Lie group, then the deformation

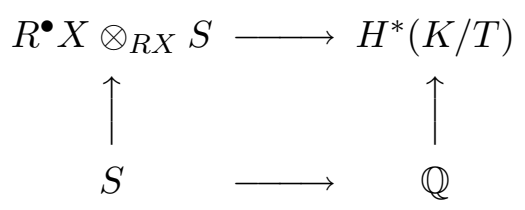

is isomorphic as a deformation to $\xi$.

Proof. This follows from the explicit construction of the versal deformation given in [8], Sec. 3. Let

$$
A_{0}=H^{*}(K / T) \cong \frac{R_{T}}{R_{K}^{+} \cdot R_{T}}=\frac{R_{T}}{\left(f_{1}, \ldots, f_{n}\right)},
$$


where the $f_{j}$ are the homogeneous generators of the $W$-invariant ring in $R_{T}$. Since $K$ is semisimple, the generators must have a degree greater than two. It follows $f_{j} \in \underline{m}_{R_{T}}^{2} \subset R_{T}$. In the following we write $R_{T}=$ $k\left[x_{1}, \ldots, x_{n}\right], \operatorname{deg} x_{i}=2$. We consider the associated exact sequence, see [8], p. 551 .

$$
0 \rightarrow \operatorname{Der}_{k}\left(A_{0}\right) \rightarrow \sum_{i=1}^{n} A_{0} \frac{\partial}{\partial x_{i}} \stackrel{\Delta}{\rightarrow} \sum_{j=1}^{n} A_{0} \frac{\partial}{\partial f_{j}} \rightarrow T_{\mathbb{Q}}^{1}\left(A_{0}\right) \rightarrow 0
$$

where $\Delta$ is the Jacobian homomorphism. It is clear that $W$ acts on all terms of this sequence such that the homomorphisms are equivariant. Now, the tangential space of $S$ can be identified with the dual of $T_{\mathbb{Q}}^{1}\left(A_{0}\right)^{W}$. By exactness one has

$$
T_{\mathbb{Q}}^{1}\left(A_{0}\right)^{W} \cong \frac{\left(\sum A_{0} \frac{\partial}{\partial f_{j}}\right)^{W}}{\operatorname{Im} \Delta \cap\left(\sum A_{0} \frac{\partial}{\partial f_{j}}\right)^{W}} .
$$

At the other hand

$$
\begin{aligned}
\operatorname{Im} \Delta \cap\left(\sum A_{0} \frac{\partial}{\partial f_{j}}\right)^{W} & =\operatorname{Im} \Delta \cap\left(\sum A_{0}^{W} \frac{\partial}{\partial f_{j}}\right) \\
& =\operatorname{Im} \Delta \cap\left(\sum A_{0}^{0} \frac{\partial}{\partial f_{j}}\right) \\
& =0
\end{aligned}
$$

as follows from the formula

$$
\Delta\left(\frac{\partial}{\partial x_{i}}\right)=\sum_{j} \overline{\left(\frac{\partial f_{j}}{\partial x_{i}}\right)} \frac{\partial}{\partial f_{j}}
$$

and $f_{j} \in \underline{m}_{R_{T}}^{2}$. Thus we have

$$
T_{\mathbb{Q}}^{1}\left(A_{0}\right)^{W}=\sum_{j=1}^{n} A_{0}^{0} \frac{\partial}{\partial f_{j}} .
$$

Now, after the recipe in [8] we choose for any basis element $\frac{\partial}{\partial f_{j}}$ a generator $t_{j}$ of degree $\operatorname{deg} t_{j}=-\operatorname{deg} \partial / \partial f_{j}=\operatorname{deg} f_{j}$ and constructing the part of $\theta_{u}$ over $S=k\left[t_{1}, \ldots, t_{n}\right] \cong R_{K}$ we get the ring

$$
A=R^{\bullet} X \otimes_{R X} S=S\left[X_{1}, \ldots, X_{n}\right] / J
$$

with $J=\left(\Phi_{1}, \ldots, \Phi_{n}\right)$ where $\Phi_{j}=f_{j}(X)-t_{j}$. Thus it follows $A \cong R_{T}$ where the homomorphism $S \rightarrow A$ is given by $t_{j} \mapsto f_{j}(X)$.

As a corollary of this result we obtain Theorem 9 .

Theorem 9. The ring homomorphism $f_{\xi}$ is an epimorphism and coincides with the ring homomorphism corresponding to the closed immersion $Y \subset V$. 
Proof. The group $K$ acts in a natural way on $X=K / T$ by left translations. This gives a continuous monoid homomorphism $\alpha: K \rightarrow \operatorname{aut}_{0}(X)$. Then $f_{\xi}=H^{*}(B \alpha)$. Thus we have a pullback diagram

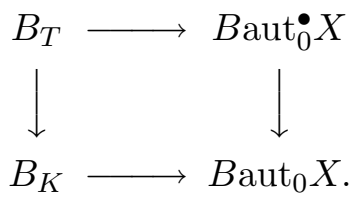

Write $E X=B$ aut $_{0}^{\bullet} X$ and $B X=B$ aut $_{0} X$. Using the Eilenberg Moore spectral sequence we get

$$
R_{T} \cong H^{*}(E X) \otimes_{R X} R_{K} .
$$

Let $f_{1}: R X \rightarrow S$ be the epimorphism corresponding to the closed immersion $Y \subset V$. Then by $W$-equivariance $f_{\xi}$ factorizes as $f_{\xi}=f_{2} \circ f_{1}$ with $f_{2}: S \rightarrow$ $R_{K}$. From the preceding result and universality of the deformation $\theta_{u}$ it follows that $f_{2}$ is an isomorphism which proves the claim.

Let now $G$ be a circle acting linearly on a $X=K / T$ with a homomorphism $\rho: G \rightarrow T$. Then the inducing homomorphism $R X \rightarrow R_{G}$ factorizes as

$$
R X \stackrel{f_{\xi}}{\longrightarrow} S \cong R_{K} \stackrel{H^{*}(B \rho)}{\longrightarrow} R_{G} .
$$

Therefore the g-points corresponding to the equivariant rational homotopy type of linear circle actions on $K / T$ lie within the subspace $Y \subset V$.

So, we get a commutative diagram

$$
\begin{array}{ccc}
O p^{\mathbb{Q}}\left(G, H^{*}(K / T)\right) & \cong & P\left(G ; H^{*}(K / T)\right) \\
\cup \uparrow & \uparrow \cup \\
O p_{\text {lin }}^{\mathbb{Q}}\left(G, H^{*}(K / T)\right) & \cong & P^{W K}\left(G ; H^{*}(K / T)\right)
\end{array}
$$

where $O p_{\text {lin }}^{\mathbb{Q}}\left(G, H^{*}(K / T)\right)$ is the set of rational equivariant homotopy classes of linear actions whereas $P^{W K}\left(G ; H^{*}(K / T)\right)$ is the fixed set of $W K$ in $P\left(G ; H^{*}(K / T)\right)$.

For to compare our results with the homotopy classification of linear circle actions on $K / T$ we conclude this note with a result giving a finer classification than equivariant rational homotopy equivalence.

Theorem 10. Let $G$ be a circle, let $X=K / T$ be the flag variety of the compact connected Lie group $K$. Suppose $\rho, \tau: G \rightarrow T \subset K$ are continuous homomorphisms and denote by $\phi_{\rho}, \phi_{\tau}$ respectively, the corresponding linear $G$-actions on $X$. Then the following assertions are equivalent:

(i) The actions $\phi_{\rho}$ and $\phi_{\tau}$ are $G$-equivariantly diffeomeorphic.

(ii) The actions $\phi_{\rho}$ and $\phi_{\tau}$ are $G$-homotopy equivalent. 
(iii) There exists an equivariant homotopy equivalence $h:\left(X, \phi_{\rho}\right) \rightarrow$ $\left(X, \phi_{\tau}\right)$.

(iv) There exists an equivariant map $h:\left(X, \phi_{\rho}\right) \rightarrow\left(X, \phi_{\tau}\right)$ which is a rational homotopy equivalence.

(v) The homomorphisms $H^{*}(B \rho ; \mathbb{Q})$ and $H^{*}(B \tau ; \mathbb{Q})$ coincide.

(vi) The representations $\rho$ and $\tau$ are conjugate by an element $w \in N_{K} T / T$ of the Weyl group.

It follows that the set of $\mathbf{g}$-points ( $\mathrm{v}$ ) gives the finest possible classification for linear action on $K / T$. For to obtain the equivariant rational homotopy classification one has to divide out the natural $\mathbb{Q}^{*}$-action. Therefore we can say that the concept of $\mathbf{g}$-points is a natural generalization of representation theory. It seems to me that this is the right place to mention that it has already been shown that the set of nonlinear g-points is non empty showing nonvoidness of our theory. Suppose a circle is acting nontrivially on a space $X$ which has the rational cohomology of $U(3) / T^{3}$. In $[\mathbf{9}]$ the authors show that up to rational cohomology there are the following fixed space configurations:

$$
\begin{array}{r}
S^{2}+S^{2}+S^{2} \\
S^{2}+S^{2}+2 p \\
S^{2}+4 p \\
6 p
\end{array}
$$

Only the first and the last configuration can be realized by linear actions. The two middle configurations are new and correspond to g-deformations of $H^{*}\left(U(3) / T^{3} ; \mathbb{Q}\right)$ which after $[\mathbf{1 3}]$ can be realized by finite $S^{1}$-complexes.

Before we begin with the proof of Thm. 10 we cite some additional information.

Lemma 14. Let $\rho, \tau: T \rightarrow H$ be homomorphisms from a torus $T$ to a compact connected Lie group $H$. If $H^{*}(B \rho ; \mathbb{Q})=H^{*}(B \tau ; \mathbb{Q})$, then $\rho$ and $\tau$ are conjugate.

For the proof see [16], Lemma 1.

Proof of Th. 10. The implications (i) $\Rightarrow$ (ii) $\Rightarrow$ (iii) $\Rightarrow$ (iv) are obvious. (iv) $\Rightarrow(\mathrm{v})$ : We consider the Borel fibrations

$$
\begin{aligned}
& \beta_{\rho}=\left\{X \rightarrow E_{G} \times_{\rho} X \rightarrow B_{G}\right\} \\
& \beta_{\tau}=\left\{X \rightarrow E_{G} \times_{\tau} X \rightarrow B_{G}\right\}
\end{aligned}
$$

which correspond to the two linear actions of $G$ on $X$. Then the map $h$ induces a rational fiber homotopy equivalence between $\beta_{\rho}$ and $\beta_{\tau}$. Denote by $f_{\rho}, f_{\tau}$ the corresponding classifying maps from $B_{G}$ in $B X=B$ aut $_{0}(X)$. By universality the corresponding rationalizations $f_{\rho, \mathbb{Q}}$ and $f_{\tau, \mathbb{Q}}$ are homotopic and therefore the induced homomorphisms $H^{*}\left(f_{\rho} ; \mathbb{Q}\right)$ and $H^{*}\left(f_{\tau} ; \mathbb{Q}\right)$ coincide. Moreover by linearity there are factorizations (up to homotopy) 
$f_{\rho}=B \alpha \circ B \rho$ and $f_{\tau}=B \alpha \circ B \tau$. Now, after Th. 9 the homomorphism $f_{\xi}=H^{*}(B \alpha ; \mathbb{Q})$ is epi. Therefore the equation $H^{*}\left(f_{\rho} ; \mathbb{Q}\right)=H^{*}\left(f_{\tau} ; \mathbb{Q}\right)$ implies $H^{*}(B \rho ; \mathbb{Q})=H^{*}(B \tau ; \mathbb{Q})$.

$(\mathrm{v}) \Rightarrow(\mathrm{vi})$ : By Lemma 14 the homomorphisms $\rho$ and $\tau$ are conjugate by an element of $K$. But since $\rho(G)$ and $\tau(G)$ are in the same maximal torus $T \subset K$, they are conjugate by an element of $W K$. (Recall that in a compact Lie group $K$ two elements $t_{1}, t_{2}$ in a fixed maximal torus $T$ are conjugate by an element of $K$ iff they are conjugate by an element of $W K$. Apply this to to the topological generators $t_{1}$ and $t_{2}$ of $\rho(G)$ and $\tau(G)$ respectively.) (vi) $\Rightarrow(\mathrm{i})$ : Let $a \in N T$ be an element such that $\tau(g)=a \rho(g) a^{-1}$. Then define $h=h_{a}:\left(K / T, \phi_{\rho}\right) \rightarrow\left(K / T, \phi_{\tau}\right)$ as $h(k T)=a k a^{-1} T$. It is then a simple calculation to show that $h$ is $G$-equivariant. It is obvious that $h$ is a diffeomorphism.

\section{References}

[1] C. Allday, On the rational homotopy of fixed point sets of torus actions, Topology, 17 (1978), 95-100, Pergamon Press.

[2] C. Allday and V. Puppe, Cohomological Methods in Transformation Groups, Cambridge studies in advanced mathematics, 32, Cambridge University Press, 1993.

[3] A.K. Bousfield and V.K.A.M. Gugenheim, On P.L.-DeRham theory and rational homotopy type, Mem. Am. Math. Soc., 179(8), Nov. 1976, Coden Mamcau.

[4] W. Bruns and J. Herzog, Cohen-Macaulay rings, Cambridge studies in advanced mathematics, 39, Cambridge University Press, 1993.

[5] T. Chang, On the number of relations in the cohomology of a fixed point set, Manuscripta Math., 18 (1976), 237-247.

[6] S. Halperin, Finiteness in the minimal models of Sullivan, T.A.M.S., 230 (1977), 173-199.

[7] V. Hauschild, The Euler characteristic as an obstruction to compact Lie group actions, T.A.M.S., 298(2) (1986), 549-578.

[8] _ Deformations and the rational homotopy of the monoid of fiber homotopy equivalences, Ill. J. Math., 37(4) (Winter 1993), 537-560.

[9] K. Hokama and S. Kono, On the fixed point set of $S^{1}$-actions on the complex flag manifolds, Math. J. Okayama Univ., 20(1) (March 1978), 1-16.

[10] W.Y. Hsiang, Cohomology Theory of Topological Transformation Groups, Ergebnisse der Math. und ihrer Grenzgebiete 85, Springer-Verlag, Berlin-Heidelberg-New York, 1975.

[11] S. Papadima, Rigidity properties of compact Lie groups modulo maximal tori, Math. Ann., 275 (1980), 637-652.

[12] V. Puppe, On a conjecture of Bredon, Manuscripta Math., 12 (1974), 11-16.

[13] Deformations of algebras and cohomology of fixed point sets, Manuscripta Math., 30 (1978), 119-136.

[14] D.S. Rim, Equivariant G-structure on versal deformation, T.A.M.S., 257 (1980), 217-226. 
[15] H. Shiga and M. Tezuka, Rational fibrations, homogeneous spaces with positive Euler characteristics and Jacobians, Ann. Inst. Fourier, 37 (1987), 81-106, Grenoble.

[16] Z. Wojtkowiak, A remark on maps between classifying spaces of compact Lie groups, Canadian Math. Bull., 31(4) (1988), 452-458.

Received March 1, 1998 and revised November 9, 1998.

\section{UNICAL}

RENDE

ITALY

E-mail address: hausch@unical.it 